"Geometrically, the line traced is a broken line which links the unit point $(1,1, \ldots, 1)$ to the origin $(0,0, \ldots, 0)$. The last segment is the part of the straight line $a_{h}=A \bar{k}_{h} / \bar{\sigma}_{h}^{2}$ inside the hypercube $0 \leq a_{h} \leq 1$ "

Bruno de Finetti

\title{
Bruno de Finetti and the Case of the Critical Line's Last Segment
}

\author{
by \\ Luca Barone \\ (Goldman Sachs International) \\ luca.barone@gs.com
}

\begin{abstract}
The anticipatory views of Bruno de Finetti on portfolio theory, set out by the author in a 1940 article, have recently been discovered by Mark Rubinstein and reviewed by Harry Markowitz. This paper analyzes the crucial parts of de Finetti's paper and discusses the controversial issue of the critical line's last segment, i.e. the segment that leads to the minimum-variance efficient portfolio. Markowitz (2006) derives the criterion for the last segment to lie on one of the boundaries of the set of legitimate portfolios in two (and $n$ ) dimensions and Pressacco (2005) shows a necessary and sufficient condition for the last segment to lie inside the legitimate set in three dimensions when the correlations are uniformly positive. This paper revises the terms of the problem and completes the analysis.
\end{abstract}

Journal of Economic Literature classification codes: C61 (Mathematical Methods and Programming - Optimization Techniques; Programming Models; Dynamic Analysis), G11 (General Financial Markets - Portfolio Choice; Investment Decisions).

Keywords: probability of default, reinsurance, mean-variance criterion, correlation, critical line algorithm.

October $9^{\text {th }}, 2006$

Paper prepared for the "Bruno de Finetti Centenary Conference", Accademia dei Lincei, Rome, November $17^{\text {th }}, 2006$. 
Bruno de Finetti and the Case of the Critical Line's Last Segment LuCA BARONE

(Goldman Sachs International)

"Bruno de Finetti Centenary Conference", Accademia dei Lincei, Rome, November $17^{\text {th }}, 2006$. 


\section{INTRODUCTION}

In 1940, Bruno de Finetti published a paper ("Il problema dei pieni") that has only recently come to the attention of English-speaking economists. The merit goes to Mark Rubinstein, who asked me to translate it into English, and to Harry Markowitz, who provided a review of de Finetti's paper. ${ }^{1}$

De Finetti' paper, which is actually a booklet (it is 88 pages long!), is complex from the very beginning, i.e. the title. It could have been translated as the "The Problem of Optimal Retention Levels in Proportional Reinsurance" but the translation would not have been faithful. Since the Italian title could have been "Il problema dei pieni assicurativi" (a little more self-explanatory for people not acquainted with the jargon of actuaries), I thought that the best way to translate it was "The problem of full-risk insurances", which retains the term ("full" for "pieni") used by de Finetti, although it is still cryptic.

"The problem of full-risk insurances" is an outstanding piece of research. This may help to explain why Franco Modigliani, when he was awarded the Nobel prize in 1985 (some months after the death of Bruno de Finetti and 5 years before Markowitz was awarded his), said he thought de Finetti would have deserved the same award.

As Rubinstein notes, de Finetti's paper was received for publication in December 1938 in response to a competition to determine "the maximum sum that an insurance company can insure retaining (full) risk exposure." ${ }^{2}$ At that time, the 32-year old Bruno de Finetti was already known as an enfant prodige (he was appointed to a chair in Mathematical Analysis when he was only 24 years old, thus becoming the youngest university professor in Italy). ${ }^{3}$

It is impressive to read the paper now and to find that so many of his ideas are become milestones in financial economics. In particular, on the basis of his 1940 paper, Bruno de Finetti can be considered one of the founders of portfolio theory.

In the first chapter of the paper, Bruno de Finetti:

1. anticipated two classical steps in capital allocation. In current jargon, the three-step process of capital allocation is made by security analysis (i.e. the input list: mean, variance and covariance of returns), portfolio selection (the efficient frontier), utility maximization (the optimal portfolio). What de Finetti called "the problem of relative full-risk insurances" is the problem of portfolio selection, while what he called "the problem of absolute full-risk insurances" is that of utility maximization (the words in square brackets are my addition, here and elsewhere):

" $\ldots$ the problem of relative full-risk insurances reduces to determining $a_{1}, a_{2}, \ldots, a_{n}$ [the portfolio weights] so that, for given $G, t[=G / \sigma]$ is maximized (i.e. determining the maximum point of $t$ for different levels $G=$ const.). Besides, put more simply, the expression for $t$ shows that, as long as we stay

\footnotetext{
${ }^{1}$ The translation of the first chapter of de Finetti's article has recently been published in the Journal of Investment Management (Joim) [see de Finetti (2006), Markowitz (2006) and Rubinstein (2006) in the References at the end of this paper]. Mark Rubinstein "learned about de Finetti's earlier work from Claudio Albanese, Professor of Mathematical Finance at Imperial College London" whom he first met at a Riskwaters Conference in London (November 1-2, 2004). "Claudio, in turn, had learned of this from Francesco Corielli, Professor of Statistics at Bocconi University, who had learned many of the details concerning de Finetti's work from Flavio Pressacco (who knew de Finetti), now Dean of Economics at the University of Udine". I gave my translation to Mark Rubinstein at the end of January 2005, when I was attending the Masters of Financial Engineering Program at Berkeley, and I received the first drafts of the articles by Rubinstein and Markowitz at the end of June 2005.

${ }^{2}$ The author described the content of his paper in a short abstract: "We examine—in its different aspects—-the problem of the risk due to hedging a set of insurances and, consequently, the problem of the retention levels, i.e., of the most efficient method to reinsure a part of such insurances to reduce the risk within the desired limits, while minimizing the loss of profit. The different aspects we consider are: the risk within a single accounting period (Chap. I), the risk for the whole existing portfolio (Chap. II), the risk related to the whole future development of the firm (Chap. III). Some concluding remarks follow (Chap. IV)."

${ }^{3}$ In that period de Finetti started to establish relationships with the most prominent mathematicians, including Andrey Kolmogorov, who is mentioned in the paper - together with Paul Lévy and Louis Bachelier - for his studies on continuous and jump stochastic processes. In particular, de Finetti refers to a paper written by Kolmogorov in 1932 in which the Russian mathematician answers to some questions on homogeneous stochastic processes that de Finetti had put forward. This interesting "correspondence" has been published in the Rendiconti of the Accademia dei Lincei.
} 
on an iso-level $G=$ const., determining the maximum of $t$ is equivalent to determining the minimum of $\sigma$, i.e. the minimum of the quadratic form $\sigma^{2}=\sum a_{h}^{2} \bar{\sigma}_{h}^{2}$.

As regards the problem of absolute full-risk insurances, whose solution requires knowledge of the function $P(t)$ [the probability of default as a function of the safety degree $t$ ], it suffices to establish the value $P^{\prime}$ for the probability of default that we consider tolerable, because then, from $P\left(t^{\prime}\right)=P^{\prime}$ we can obtain the corresponding value $t^{\prime}$ of $t$, and finally, denoting by $t(G)$ the maximum of $t$ on the iso-level $G$, we can determine from $t\left(G^{\prime}\right)=t^{\prime}$ the corresponding value $G^{\prime}$ of $G$. Then, the solution of the problem of absolute full-risk insurances is that of the problem of the relative full-risk insurances on isolevel $G=G^{\prime}$." (Joim, p. 23)

2. worked out a special case of the Kuhn-Tucker theorem, with upper and lower bounds on variables but no equality constraints (Joim, p. 24): ${ }^{4}$

"... what remains important is to see what is the role played by the constraints $0 \leq a_{k} \leq 1$, which we have not yet considered. It would be easy to see, immediately and directly, even in the case where the constant $A$ is not so small as to make all the $a_{h} \leq 1$, that the solution is the same as before but now it is necessary to set equal to 1 all the $a_{h}$ which would be larger than $1 . "$

3. "worked out an early version of the critical line algorithm, the numerical method used to solve the portfolio selection problem". The critical-line algorithm is described both for the nocorrelation case (Joim, p. 25) and for the correlation case (Joim, p. 30):

"Geometrically, the line traced is a broken line which links the unit point $(1,1, \ldots, 1)$ to the origin $(0$, $0, \ldots, 0)$. The last segment is the part of the straight line $a_{h}=A \bar{k}_{h} / \sigma_{h}^{2}$ inside the hypercube $0 \leq a_{h} \leq 1$ "

"Let us now consider the general case in which the risks are instead correlated. It is not difficult to extend the treatment applied so far to such a case ... The geometric interpretation given for the nocorrelation case still holds, since the line of 'optimal' points of $\sigma$ for given $G$ is a continuous broken line which links the starting point $\left(\bar{C}_{1}, \bar{C}_{2}, \ldots, \bar{C}_{n}\right)$ to the origin $(0,0, \ldots, 0)$."

4. was one of the first to emphasize the importance of covariance and correlation in capital allocation problems:

"The extension, which seems so obvious to me, of the previous considerations on risk to the case of correlated events is almost never discussed in the research on this subject (to be more precise, I remember having seen it only in a paper by Dubois ... who however didn't address the problem of full-risk insurances)" (Joim, p. 37)

5. showed that he was aware of bounds on negative correlation coefficients (Joim, p. 38) [he refers to another paper ("About correlation"), that he had published in 1937]:

“... we can always represent $n$ random numbers by $n$ vectors having a modulus equal to their standard deviations and forming - two by two - an angle whose cosine (in magnitude and sign) is measured by the correlation coefficient. Now, $n$ vectors can surely be orthogonal among each other (no-correlation case), or can form acute angles (positive correlation), but it is impossible that they form angles which are all obtuse (over a certain angle limit). For example, three vectors can form, among one another, angles of at most $120^{\circ}$. In general $n$ vectors can form angles whose cosine is at most equal to $-1 /(n-1)$. And, therefore, this is the maximum negative correlation coefficient that we can simultaneously observe among $n$ random numbers."

6. defined and analyzed the variable $t$ (called the safety degree, Joim p. 26) which corresponds to the "Sharpe ratio" when the risk-free interest rates are zero. Besides, in its context, $t$ has the meaning of KMV distance to default;

"... the probability of default will only depend on $t$ [distance to default] and precisely it will be represented by a decreasing function of $t, P(t)$ that, in particular, is

\footnotetext{
${ }^{4}$ The Kuhn-Tucker conditions are the single most important analytical result in nonlinear programming. For a good illustration see Chiang (1974, pp. 704-13).
} 


$$
P(t)=\frac{1}{\sqrt{2 \pi}} \int_{t}^{\infty} e^{-1 / 2 x^{2}} d x
$$

if the distribution is Gaussian." (Joim, p. 23)

7. sketched what can be considered an embryo of Nash equilibrium theory (Joim, p. 41):

"If we consider several insurers who offer reinsurance to one another and everyone of these behaves - when determining the levels of full-risk insurances - in the optimal way suggested by our preceding results, that is each one seeks the solution which is the most advantageous for his own ends, it cannot taken for granted that they will actually succeed in reaching their aim [This statement is related, as a particular case, to my preceding considerations intended to disprove the fundamental principle of a liberal economy, according to which the free play of single egoisms would produce a collective "optimum" ...]. Therefore, we will now try to see whether - addressing the problem simultaneously, rather than unilaterally, and using the conclusions of such a study to formulate a mutual convention rather than many unilateral decisions - it is possible to establish a different criterion which, for all and everyone, is more advantageous than the one we found before."

\section{HISTORICAL NOTE}

Only a few of the 283 works by de Finetti [listed by Daboni (1986)] are not written in Italian: 20 in English and 17 in French. Some of them have been translated: 13 into English, 2 into French, and 1 into German (the German translation refers to an article on the reinsurance problem written by de Finetti in 1942).

After the second world war, de Finetti left Assicurazioni Generali and, as Rubinstein writes in his Introduction, "became absorbed by his other work in mathematics".

It is not easy to find references to his 1940 paper. An exception is the "Notes on Foreign Actuarial Journals" in the Journal of the Institute of Actuaries (1947), which laconically refers to:

"An algebraical study of the risk problem for a single case, for the whole existing portfolio, and for the future of the whole undertaking"

An important acknowledgement comes from Hans Bühlmann (1970), whose book is reviewed in the Transactions of the Society of Actuaries:

"In Chapter 5, "Retention and Reserves," Bühlmann discusses the relative and absolute retention limits for both proportional and nonproportional reinsurance. In the section on relative retention he gives a method of de Finetti from a 1940 Italian paper. Although these ideas appear to be of great use and are known widely in Europe, we are indebted to Bühlmann for making them available in English.”

Bühlmann worked at Swiss Reinsurance Company before taking the chair in actuarial mathematics at the Federal Institute of Technology in Zurich. This may explain the reference to de Finetti's paper in the forward to a paper by Swiss Re (2003): ${ }^{5}$

"The publication is based on Bruno de Finetti's work on the establishment of optimal proportional retentions which was published in a 1940 article entitled "Il problema dei pieni". ... Because de Finetti's work remained virtually unknown outside universities, actuaries and other professionals are still to a large extent unfamiliar with practicable rules deriving from his observations. For this reason, even readers with knowledge of risk theory will also find something new in the following work."

De Finetti's contribution to portfolio theory was also noted by Pressacco (1986):

\footnotetext{
${ }^{5}$ The forward refers to a paper by Hans Schmitter (2003). The publication includes "an Excel file developed by Pamela Hall which features tools to determine useable retentions". See www.swissre.com.
} 
"The name of H. Markowitz (1952) is famous all over the world for his mean variance approach to the portfolio selection problem, a milestone in the analysis of relevant economic problems under uncertainty. It is perhaps surprising to find that more than ten years earlier B. de Finetti (1940) used the same approach to study a key problem in proportional reinsurance: the optimal retention problem."

and stressed by Daboni and Pressacco (1987):

“... de Finetti's paper is to be seen as an early anticipation of H. Markowitz's (Markowitz, 1952) well known two stage mean variance approach to the portfolio selection problem."

For historical notes on the genesis of portfolio theory, see Markowitz (1999) and Rubinstein (2002). ${ }^{6}$

\section{GEOMETRIC VIEW}

The giant upon whose shoulders de Finetti's solution to the reinsurance problem stands is a shy and modest Italian, born in Turin, and considered the greatest mathematician of the eighteenth century: Giuseppe Luigi Lagrangia, better known as Joseph-Louis Lagrange. Even if de Finetti does not mention him, the first step in solving the reinsurance problem was taken by using Lagrange multipliers, the powerful tool introduced in 1759 by the 23 -year old mathematician. ${ }^{7}$

The reinsurance problem studied by de Finetti (1940) is very similar to, but formally different from, the portfolio selection problem analyzed by Markowitz (1952). In both problems the goal is to minimize the variance of a portfolio's return, for a given level of expected return, subject to some linear constraints (a quadratic programming problem).

An analytical comparison of the two approaches is shown in the next section. For the time being it is sufficient to state that Markowitz studies how to select efficient portfolios by investing a unit of capital, while de Finetti considers a given portfolio and studies how to revise its weights, by selling (i.e. reinsuring) some of the securities (i.e. insurance policies), in order to obtain efficient portfolios. ${ }^{8}$ Therefore, both de Finetti and Markowitz study how to find the optimal weights $\left(a_{1}, a_{2}, \ldots, a_{n}\right.$ in de Finetti's notation, $X_{1}, X_{2}, \ldots, X_{n}$ in Markowitz's notation) of efficient portfolios.

Under the hypotheses that we will set out later, the symbol $E$ used by Markowitz to denote the portfolio's expected return is equal to the symbol $G$ used by de Finetti. We are now ready to understand the words (and symbols) used by de Finetti in setting up his reinsurance problem:

"... the problem of relative full-risk insurances [i.e., the problem of computing the set of mean-variance efficient portfolios] reduces to determining [the retention levels] $a_{1}, a_{2}, \ldots, a_{n}$ so that, for a given [expected return] $G, t[=G / \sigma]$ is maximized (i.e. determining the maximum point of $t$ for different levels $G=$ const.). Besides, put more simply, the expression for $t$ shows that, as long as we stay on an iso-level $G=$ const., determining the maximum of $t$ is equivalent to determining the minimum of $\sigma$, i.e. the minimum of the quadratic form $\sigma^{2}=\sum a_{h}^{2} \sigma_{h}^{2}$ [the no-correlation case]". (Joim, p. 23)

Markowitz (2006) approves the de Finetti analysis for the no-correlation case ("For the case of uncorrelated risks, de Finetti solved the problem of computing the set of mean-variance efficient portfolios."). Therefore, we could skip this case and focus on correlated risks. However, since it is instructive to see how de Finetti solved the problem when risks are not correlated, we will look at the geometric solution of the no-correlation case, in two and three dimensions, before examining the correlation case.

\footnotetext{
${ }^{6}$ See also Markowitz (1987, pp. 36-40) and Markowitz (2006, pp. 16-7).

${ }^{7}$ See Lagrange (1759). A good description of the Lagrange-multiplier method is in Chiang (1974, pp. 376-9). Bruno de Finetti $(1937 \mathrm{a}, \mathrm{b})$ wrote two articles on free and constrained optimization problems before writing the 1940 paper.

${ }^{8}$ The securities considered by de Finetti are life insurance policies: “... we intend to discuss the question in the case of life insurances (which - because of their intrinsic characteristics - will lead to further considerations) ...”.
} 


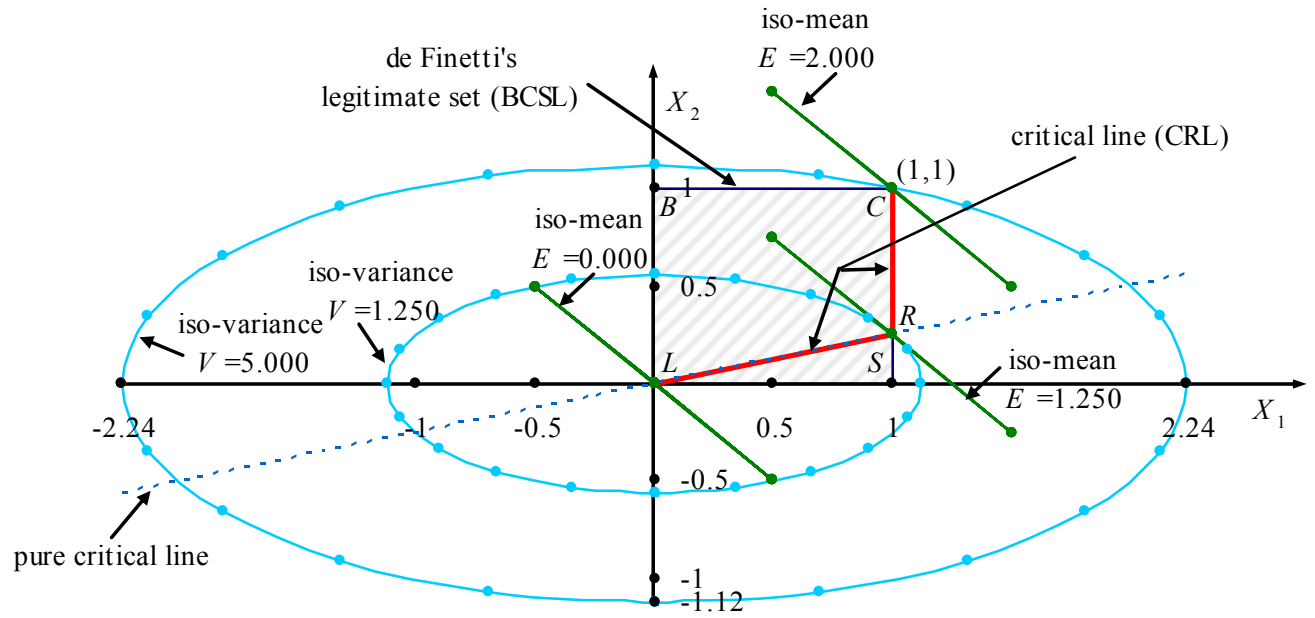

Figure 1 Efficient portfolios in the space $\left\{X_{1}, X_{2}\right\}: \mu_{1}=1, \mu_{2}=1, \sigma_{1}=1, \sigma_{2}=2, \rho_{12}=0.0$

\section{Uncorrelated Assets}

Two Assets

Let us consider the numerical example given by Markowitz (2006), where the rates of return of two securities have means equal to $1\left(\mu_{1}=\mu_{2}=1\right)$ and standard deviations equal to 1 and 2 , respectively $\left(\sigma_{1}=1, \sigma_{2}=2\right)$. Differently from Markowitz, let us initially suppose that the returns are uncorrelated $\left(\rho_{12}=0\right)$. The elements of the problem are reported in Figure 1 .

The portfolio weights cannot be either less than 0 (because policies cannot be over-reinsured) or more than 1 (policy re-issue is not allowed). Therefore, a portfolio is legitimate if and only if it lies on or in the square BCSL. This area is referred to as the set of legitimate portfolios. ${ }^{9}$

The iso-mean lines, i.e. the lines made up of portfolios whose returns have a mean equal to a given level $E$, are represented by straight lines: “... the iso-mean lines form a system of parallel lines, with a direction of increasing $E$.." [Markowitz (1976), p. 135].

The iso-variances curves, i.e. the curves made up of portfolios whose returns have a variance equal to a given level $V$, are represented by ellipses: "All these ellipses have the same center, the same orientation, and the same ratio of longest diameter to shortest diameter" [Markowitz (1976), p. 136]. In the example, the diameters of the ellipses coincide with the axes because the correlation is null. The longest diameters lie on the axis for $X_{1}$, because this is the axis that measures the weight of the policy with the lowest variance: for the portfolio to have a certain variance, $V$, the weight of $X_{1}$ needs to be bigger than the weight of $X_{2}$, the policy with the highest variance. If the variances of the two policies were the same, the ellipses would be circles.

An efficient portfolio is a legitimate portfolio that offers the highest mean, $E$, for a given variance, $V$, as well as the lowest variance, $V$, for given mean, $E$. To select efficient portfolios, let us first find "the locus of all tangencies between iso-mean lines and iso-variance curves" [Markowitz (1976), p. 139]. The line connecting all these points will be called the pure critical line. Since it does not take into account the bounds of the variables, it represents the solution to a constrained optimization problem.

\footnotetext{
${ }^{9}$ Roy (1952) allows short sales. Therefore, his legitimate set is given by the square ACFG and the critical line is CRH.
} 


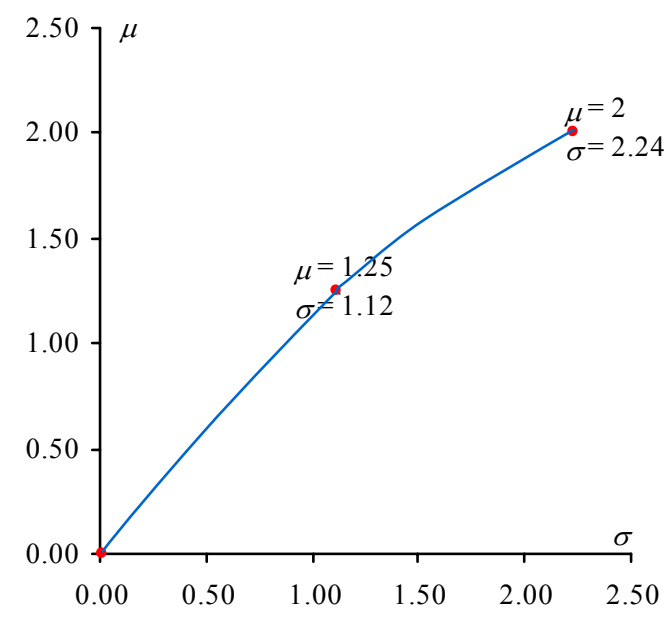

Figure 2 Efficient frontier

The solution to the quadratic programming problem is given by the broken line $C R L$ detected by Markowitz's (1956) critical line algorithm (CLA). The points $C, R, L$ are called corner portfolios. The construction of the broken line is explained by Markowitz (1974, p. 151) in the following terms:

"The relationship between critical lines and efficient portfolios can be described by a simple device. Imagine the critical lines as railroad tracks. Imagine, further, that a passenger boards a train at $\underline{X}[\underline{X}$ is the legitimate portfolio with variance smaller than that of any other portfolio] and travels in the direction of increasing expected return. The first time his train crosses another track - as soon as the first critical line intersects a second critical line - the passenger transfers to the new track, the new critical line, and again travels in the direction of increasing $E$ Again he reaches another track, an intersection of critical lines, and again he transfers to the new, continuing in the direction of increasing $E$. This continues until the passenger reaches $\bar{X}$ with maximum $E$. There his journey ends.

Every point through which the traveler passes on his journey represents an efficient portfolio. Conversely, every efficient portfolio was reached en route. ..."

In the critical line algorithm, the direction actually followed by the passenger is reversed. The passenger starts from $\bar{X}$ and reaches $\underline{X}$. To detect the starting point $\bar{X}$, i.e. the legitimate portfolio with a higher expected return than that of any other portfolio, the CLA solves a linear programming problem by making use of George Dantzig's (1951) simplex algorithm. In solving the reinsurance problem, the simplex method is not needed, since the starting point is known (it is given by the portfolio that is fully invested in all the available policies). However, the solution of the reinsurance problem will not necessarily represent the set of all the efficient portfolios, i.e. the efficient frontier, since we do not know if the initial portfolio is efficient or not.

The solution traced by the CLA in the space of the portfolio weights $\left\{X_{1}, X_{2}\right\}$ can be projected in the space $\{E, V\}$ or $\{E, \sigma\}$ to derive the better known shape of the efficient frontier [Markowitz (1974, pp. 152-3)]:

"As long as our passenger moves along a single critical line, the relationship between expected return and variance of return is represented by a parabola ...

The curve relating efficient standard deviation to efficient expected return has properties similar to the curve relating $E$ and $V$. The chief difference is that the former is principally made of segments of hyperbolas rather than parabolas ..."

The efficient frontier for the example we have examined is reported in Figure 2, where corner portfolios are highlighted by markers, and in Table 1. 
TABLE 1 Efficient portfolios in the space $\left\{X_{1}, X_{2}\right\}: \mu_{1}=1, \mu_{2}=1, \sigma_{1}^{2}=1, \sigma_{2}^{2}=4, \rho_{12}=0.0$

\begin{tabular}{|c|c|c|c|c|c|c|c|c|}
\hline \multirow{2}{*}{$\begin{array}{l}\text { Corner } \\
\text { portfolio } \\
(\#)\end{array}$} & \multirow{2}{*}{$\begin{array}{l}\text { Expected rate } \\
\text { of return } \\
(E)\end{array}$} & \multirow{2}{*}{$\begin{array}{l}\text { Standard } \\
\text { deviation } \\
(\sqrt{V})\end{array}$} & \multirow{2}{*}{$\begin{array}{l}\text { Lagrange } \\
\text { multiplier } \\
\qquad\left(\lambda_{E}\right)\end{array}$} & \multicolumn{3}{|c|}{$\begin{array}{c}\text { Function } \\
V=a_{0}+a_{1} E+a_{2} E^{2}\end{array}$} & \multicolumn{2}{|c|}{$\begin{array}{c}\text { Corner portfolio's } \\
\text { weights }\end{array}$} \\
\hline & & & & $a_{0}$ & $a_{1}$ & $a_{2}$ & $X_{1}$ & $X_{2}$ \\
\hline 1. & 2.000 & 2.236 & 4.000 & & & & 1.000 & 1.000 \\
\hline 2. & 1.250 & 1.118 & 1.000 & 5.000 & -8.000 & 4.000 & 1.000 & 0.250 \\
\hline 3. & 0.000 & 0.000 & 0.000 & 0.000 & 0.000 & 0.800 & 0.000 & 0.000 \\
\hline
\end{tabular}

Note: The table shows the output of the VBA portfolio selection program reported in Markowitz and Todd (2000).

TABLE 2 Efficient portfolios in the space $\left\{X_{1}, X_{2}, X_{3}\right\}: \mu_{1}=\mu_{2}=\mu_{3}=1, \sigma_{1}=\sigma_{3}=1, \sigma_{2}=2, \rho_{12}=\rho_{13}=\rho_{23}=0$

\begin{tabular}{|c|c|c|c|c|c|c|c|c|c|}
\hline \multirow{2}{*}{$\begin{array}{l}\text { Corner } \\
\text { portfolio } \\
(\#)\end{array}$} & \multirow{2}{*}{$\begin{array}{l}\text { Expected } \\
\text { rate of return } \\
(E)\end{array}$} & \multirow{2}{*}{$\begin{array}{l}\text { Standard } \\
\text { deviation } \\
(\sqrt{V})\end{array}$} & \multirow{2}{*}{$\begin{array}{l}\text { Lagrange } \\
\text { multiplier } \\
\qquad\left(\lambda_{E}\right)\end{array}$} & \multicolumn{3}{|c|}{$\begin{array}{c}\text { Function } \\
V=a_{0}+a_{1} E+a_{2} E^{2}\end{array}$} & \multicolumn{3}{|c|}{$\begin{array}{l}\text { Corner portfolio's } \\
\text { weights }\end{array}$} \\
\hline & & & & $a_{0}$ & $a_{1}$ & $a_{2}$ & $X_{1}$ & $X_{2}$ & $X_{3}$ \\
\hline 1.000 & 3.000 & 2.449 & 4.000 & & & & 1.000 & 1.000 & 1.000 \\
\hline 2.000 & 2.250 & 1.500 & 1.000 & 18.000 & -16.000 & 4.000 & 1.000 & 0.250 & 1.000 \\
\hline 3.000 & 0.000 & 0.000 & 0.000 & 0.000 & 0.000 & 0.444 & 0.000 & 0.000 & 0.000 \\
\hline
\end{tabular}

Note: The table shows the output of the VBA portfolio selection program reported in Markowitz and Todd (2000).

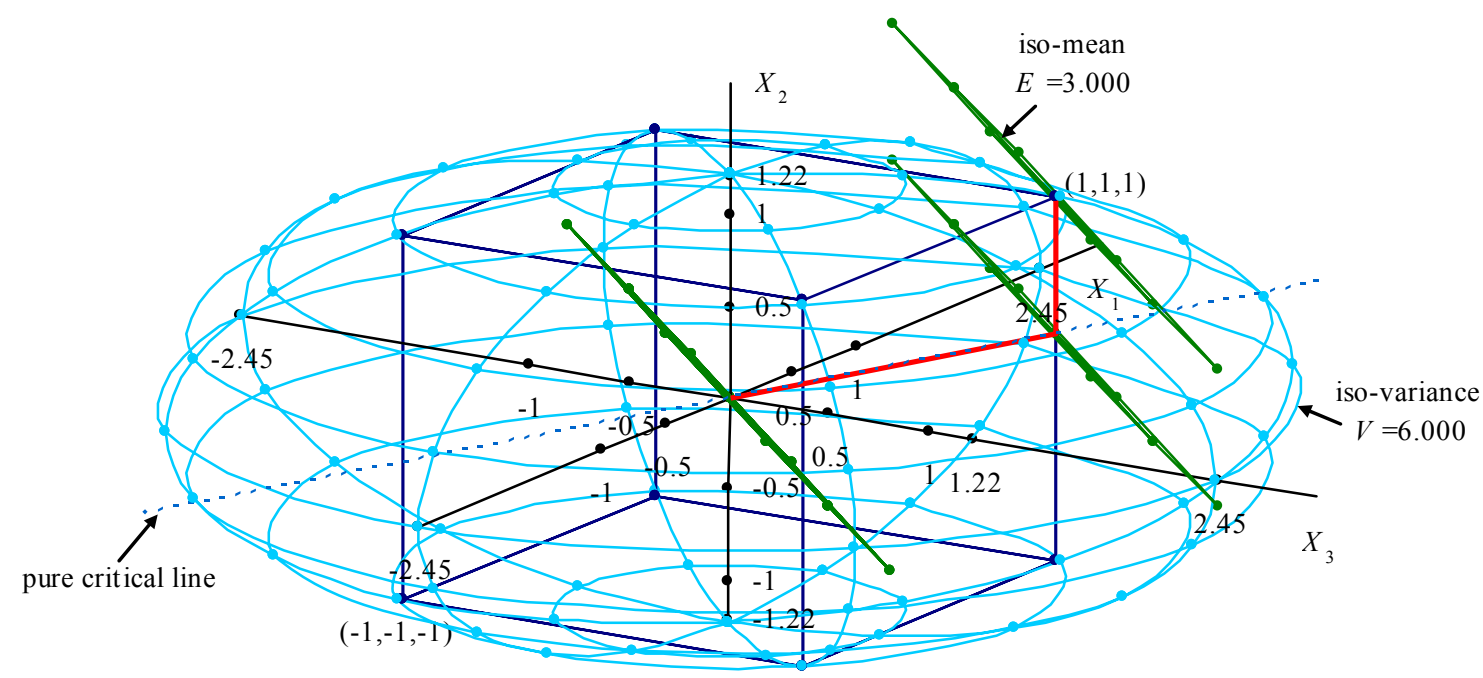

Figure 3 Efficient portfolios in the space $\left\{X_{1}, X_{2}, X_{3}\right\}: \mu_{1}=\mu_{2}=\mu_{3}=1, \sigma_{1}=\sigma_{3}=1, \sigma_{2}=2, \rho_{12}=\rho_{13}=\rho_{23}=0$

\section{Three Assets}

Let us now consider a portfolio with three uncorrelated policies. The rates of return have means equal to $1\left(\mu_{1}=\mu_{2}=\mu_{3}=1\right)$ and standard deviations equal to 1,2 and 1 , respectively $\left(\sigma_{1}=\sigma_{3}=1, \sigma_{2}=2\right)$.

Since the variances are not all equal, iso-variances are ellipsoids, i.e. spheres which are stretched somewhere and compressed elsewhere. For instance, policies 1 and 3, which have the lowest variances, must receive more weight for the portfolio's variance to reach a certain level, $V$. Therefore, the ellipsoid appears stretched along the $X_{1}$ and $X_{3}$ axes (Figure 3). ${ }^{10}$

\footnotetext{
${ }^{10}$ The figure has been rotated clockwise. The rotation angles with respect to the three axes are, respectively, $10^{\circ}, 59^{\circ}$, and $16^{\circ}$.
} 


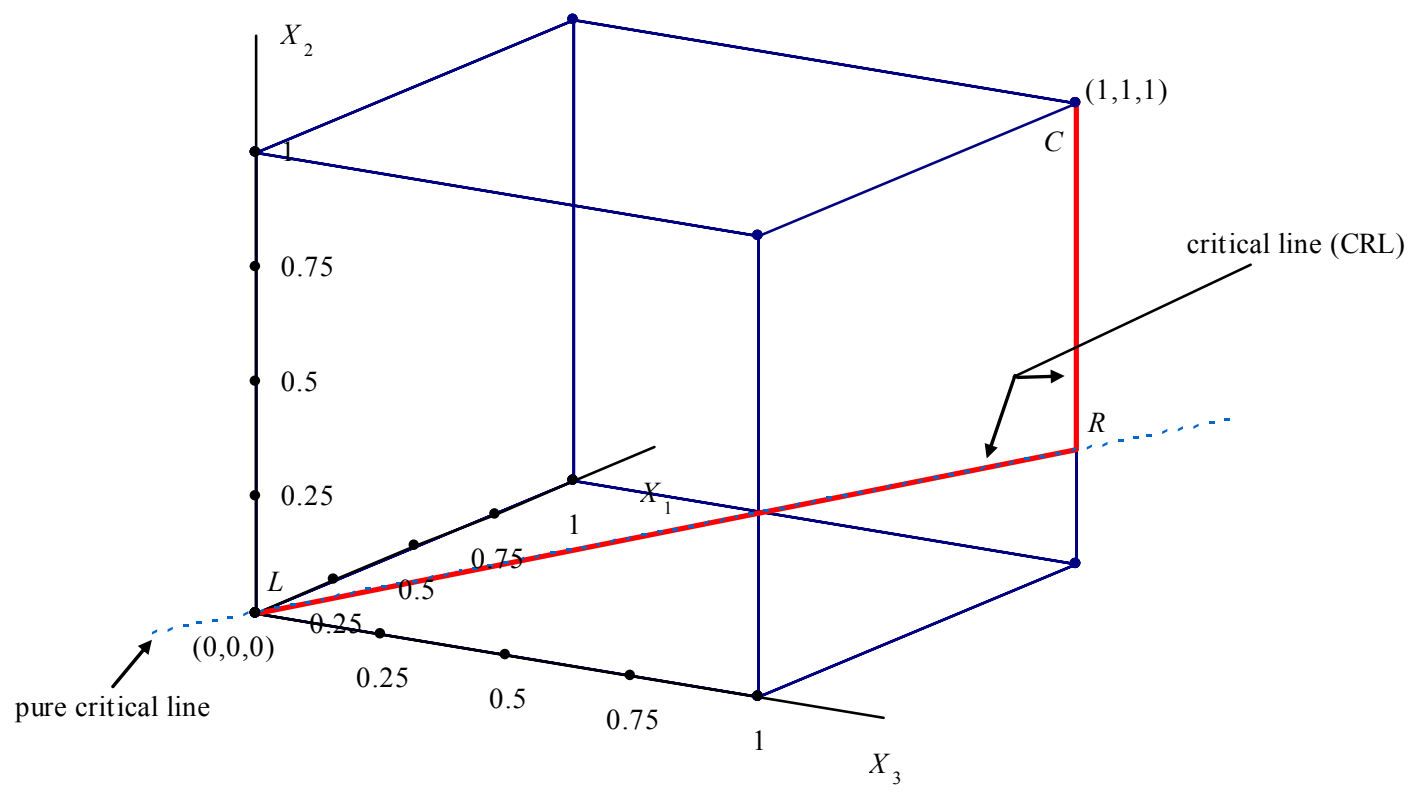

Figure 4 The critical line in 3D (no-correlation case)

Vice versa, policy 1, which has the highest variance, must receive less weight for the portfolio variance to reach the same level, $V$. Therefore, the ellipsoid appears compressed along the $X_{2}$ axis.

The set of legitimate portfolios is given by the cube shown in Figure 4. The solution must be found in or on the cube.

What does de Finetti say for the no-correlation case? He describes the solution both analytically and geometrically ("perhaps the following argument, which allows us to see the path along which it is more advantageous to proceed, as we decrease the risk by increasing the reinsurance, will be more illuminating"). First of all, he defines the iso-means (hyperplanes) and the iso-variances (ellipsoids):

“... the loci of points with equal $G[E]$ form a system of parallel hyperplanes, while the loci of points with equal $\sigma$ are ellipsoids (concentric, homothetic, centered at the origin and having as principal axes the axes $\left.a_{1}, \ldots, a_{n}\left[X_{1}, \ldots, X_{n}\right]\right)$."

Then, he solves the constrained optimization problem by using Lagrange multipliers:

"If we do not consider the constraints $0 \leq a_{h} \leq 1\left[0 \leq X_{i} \leq 1\right]$, the solution of the analytical problem is given by the straight line (passing through the origin) [the pure critical line] which is the locus of all tangencies between ellipsoids and hyperplanes."

Finally (this is the part where he works out a special case of the Kuhn-Tucker theorem),

"... what remains important is to see what is the role played by the constraints $0 \leq a_{k} \leq 1\left[0 \leq X_{i} \leq 1\right]$, that we didn't yet consider. It would be easy to see, immediately and directly, ... that the solution is the same as before but now it is necessary to set equal to 1 all the $a_{h}\left[X_{i}\right]$ which would be larger than $1 . "$

In conclusion:

"Geometrically, the line traced is a broken line which links the unit point $(1,1, \ldots, 1)$ to the origin $(0,0$, $\ldots, 0)$. The last segment is the part of the straight line $a_{h}=A \bar{k}_{h} / \bar{\sigma}_{h}^{2}$ [the pure critical line $\left.\left.X_{i}=\lambda_{E} \mu_{i} / \sigma_{i}^{2}\right]\right]$ inside the hypercube $0 \leq a_{h} \leq 1\left[0 \leq X_{i} \leq 1\right]$ while the other segments are the (orthogonal) projections of parts of the same line on the faces with $n-1, n-2, \ldots, 3,2$ dimensions, respectively, and finally on the edge, as the straight line and the projections exit the hypercube (speaking here in a retrograde sense with respect to the preceding description)." 
Therefore, the pure critical line is given by the equation $X_{i}=\lambda_{E} \mu_{i} / \sigma_{i}^{2}$, where $X_{i}$ is a function of $\lambda_{E}$. We can now check - for the example we have considered so far - that the last segment of the critical line does actually lie on the pure critical line. In Table 2 the $\lambda_{E}$ for the penultimate and ultimate corner portfolios is equal to 1 and 0 , respectively. By recalling that $\mu_{1}=\mu_{2}=\mu_{3}=1, \sigma_{1}=\sigma_{3}=1, \sigma_{2}=2$, the last segment is uniquely defined by the points $\left(X_{1}=1, X_{2}=0.25, X_{3}=1\right)$ and $\left(X_{1}=0, X_{2}=0, X_{3}=\right.$ 0 ), which actually lie on the pure critical line.

Finally, it is worth noting that, after reaching the penultimate corner portfolio, all the contracts must be reinsured in equal proportion, by maintaining the ratio $X_{1} \sigma_{1}^{2} / \mu_{1}=X_{2} \sigma_{2}^{2} / \mu_{2}=X_{3} \sigma_{3}^{2} / \mu_{3}=$ $\lambda_{E}$.

\section{Four Assets}

To fully understand de Finetti's safety-degree curve (Figure 1, p. 27 in Joim), which can be considered his version of the $\mu$ vs. $\sigma$ efficient frontier, it is necessary to remove the hypothesis (made so far) that the expected return on the completely reinsured portfolio is null. When we remove this hypothesis, the reinsurance problem is no longer equal to the portfolio selection problem.

To solve the general reinsurance problem by the critical line algorithm, we have to consider a fourth asset, which is just the original portfolio held by the insurer. In the new problem: ${ }^{11}$

1. the expected rate of return on the original portfolio may be different from the sum of the expected rates of return on the three policies;

2. the fourth row and the fourth column of the new variance-covariance matrix must be constructed in the way shown in the Appendix (page 26);

3. the weight of the fourth asset must always to be equal to 1 , while the weights of the first three assets must lie in the range between -1 and 0 .

In his Figure 1 de Finetti shows the behavior of the safety degree, $E / \sqrt{V}$, as a function of $\lambda_{E}$. Since the critical line algorithm starts from $\lambda_{E}=+\infty$ (no reinsurance) to end with $\lambda_{E}=0$ (total reinsurance), the chart has to be read from right to left if we want to see how the safety degree changes when we move from more risky to less risky portfolios.

Let $v_{0}$ and $\sigma_{0}$ be, respectively, the mean and standard deviation of the rate of return on the original portfolio held by the insurer. Besides, let us suppose we have numbered the policies so that $\left(\mu_{1} /\right.$ $\left.\sigma_{1}\right)<\left(\mu_{2} / \sigma_{2}\right)<\left(\mu_{3} / \sigma_{3}\right)$. In other terms, the first contract - the one with the lowest reward-tovariability ratio (or safety degree) - is the first to be reinsured.

We have to distinguish between three / four cases that are characterized, respectively, by:

1. $v_{0}>\left(\mu_{1}+\mu_{2}+\mu_{2}\right)$, when the insurer earns a positive amount on the completely reinsured portfolio;

2. $v_{0}=\left(\mu_{1}+\mu_{2}+\mu_{2}\right)$, when the insurer breaks even if he completely reinsures his portfolio;

3. $v_{0}<\left(\mu_{1}+\mu_{2}+\mu_{2}\right)$, when the insurer sustains a loss if he completely reinsures his portfolio, and:

a. $\left(\mu_{1} / \sigma_{1}\right)<\left(v_{0} / \sigma_{0}\right)$;

b. $\left(\mu_{1} / \sigma_{1}\right)>\left(v_{0} / \sigma_{0}\right)$.

The safety degree charts corresponding to the above hypotheses are reported in Figure 5, which replicates de Finetti's Figure 1. In Figure 5a the safety degree increases more and more (from right to left) as the reinsurance increases. In Figure $5 b$ "the safety degree stops growing from the point where all the contracts start to be reinsured" that is when the critical line's last segment is reached. In Figure $5 \mathrm{c}$ the safety degree is improved by reinsurance until a certain point, after which it decreases. In Figure $5 d$ "the reinsurance - whatever is its method and extent - can only decrease the safety degree".

\footnotetext{
${ }^{11}$ The proof of the equivalence between the two problems when transaction costs are null is shown in the Appendix (page 26).
} 


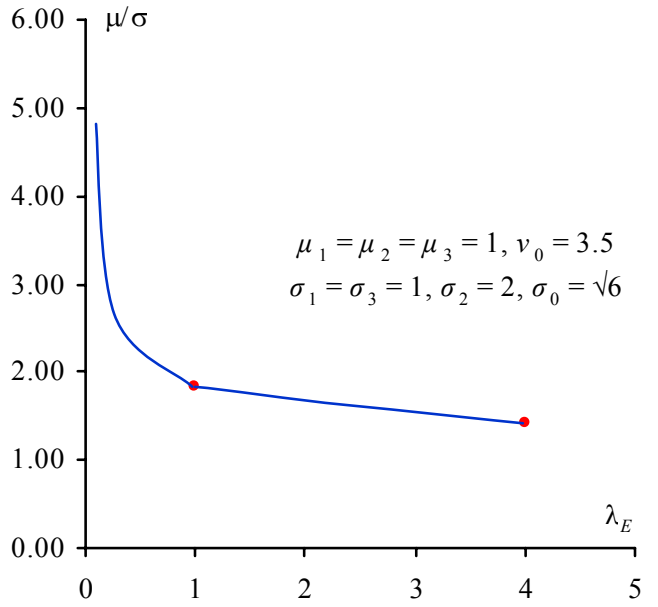

(a)

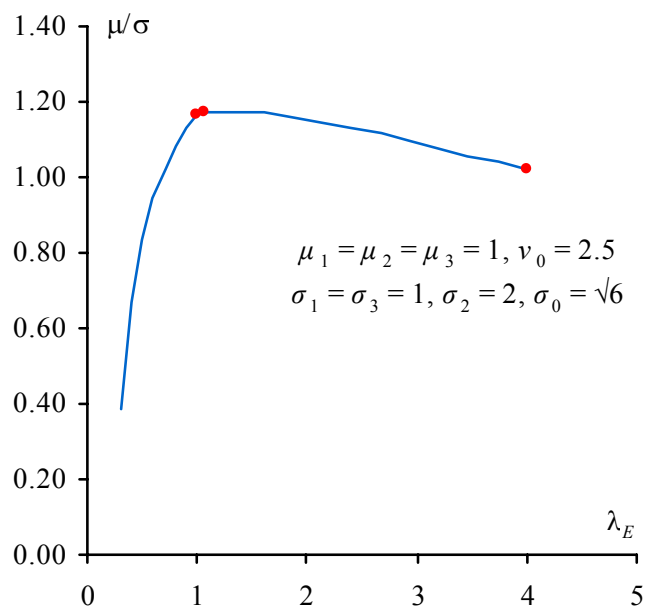

(c)

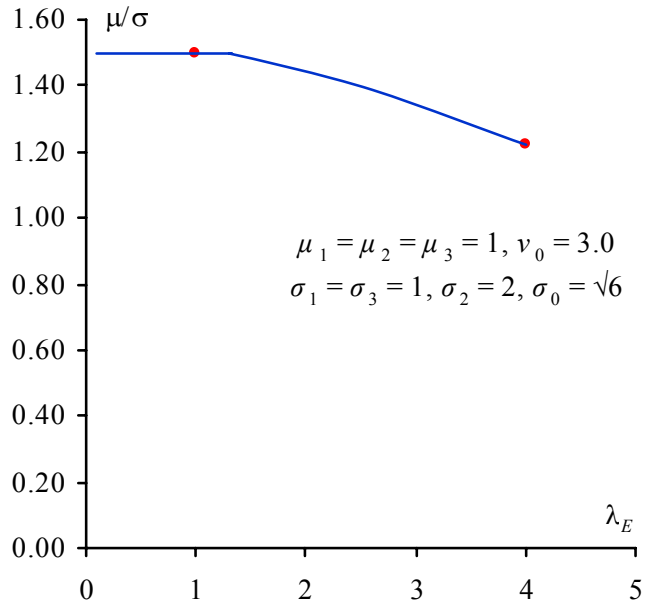

(b)

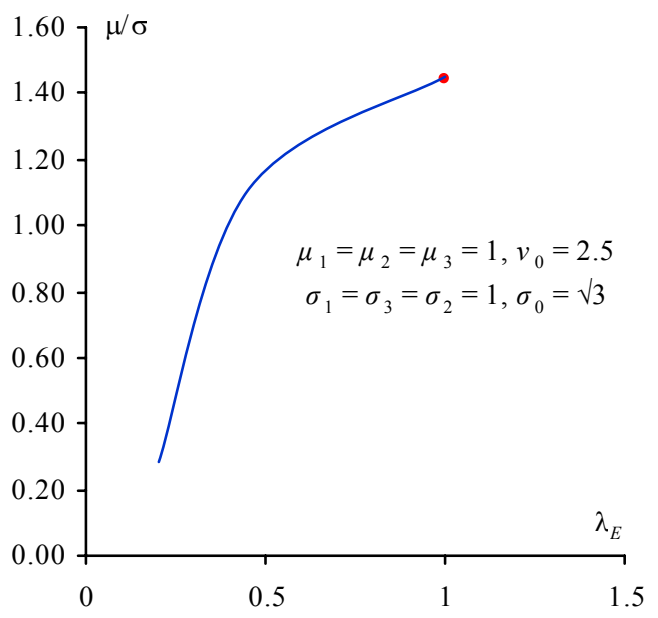

(d)

Figure 5 The safety-degree curve

\section{Correlated Assets}

Two Assets

Let us now consider two correlated assets, whose rates of return have means $\mu_{1}=\mu_{2}=1$ and standard deviations $\sigma_{1}=1, \sigma_{2}=\sqrt{1.5}$. The correlation coefficient is $\rho_{12}=0.6$. The geometric solution, reported in Figure 6, can be compared with that already shown for the no-correlation case (Figure 1).

The most striking difference between the two figures is given by the iso-variance curves, whose diagonals no longer lie on the main axes. Since the correlation is positive, when the signs of both variables are equal (both positive or both negative) the iso-variance curves are closer to the main axes than in the case when the signs are different. Positive correlation strengthens the effect of the variance of each asset on the portfolio's variance, thus allowing small weights to produce a given level of portfolio variance, but only if the weights have the same sign.

The reverse is true for negative correlation. Therefore, the orientation of the system of ellipses depends on correlation. When correlation is positive, the orientation is negative, and vice versa. 


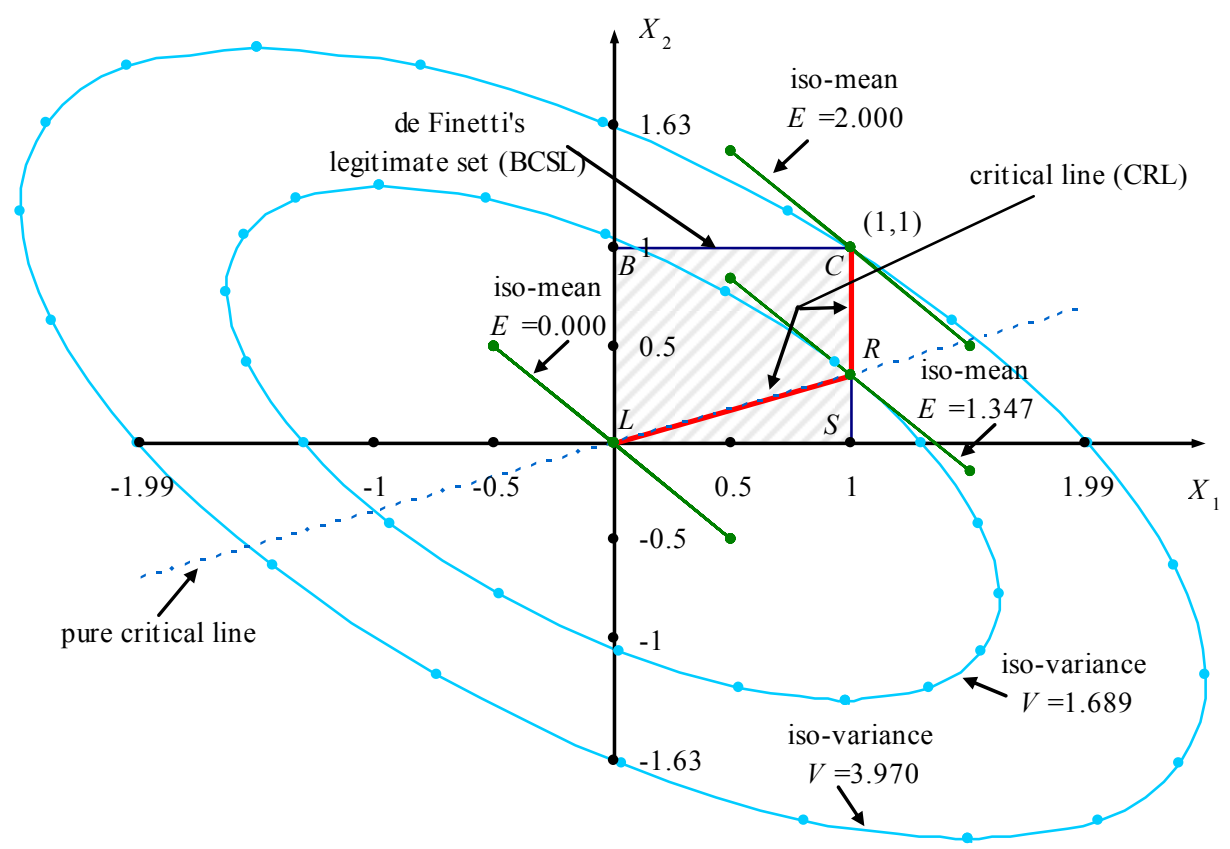

Figure 6 Efficient portfolios in the space $\left\{X_{1}, X_{2}\right\}: \mu_{1}=1, \mu_{2}=1, \sigma_{1}=1, \sigma_{2}=\sqrt{1.5}, \rho_{12}=0.6$

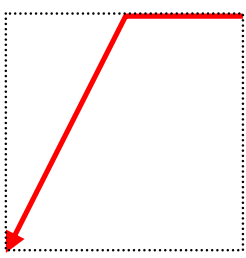

(a)

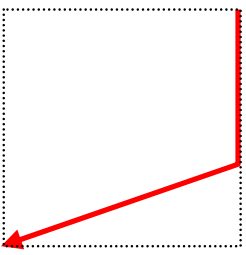

(b)

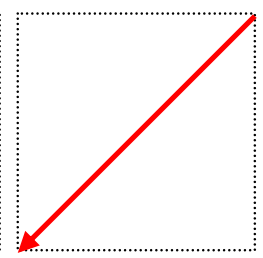

(c)

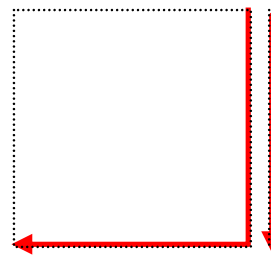

(d)

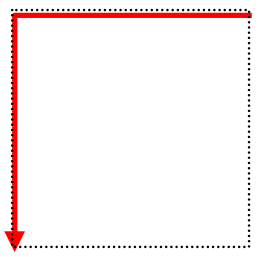

(e)

Figure 7 Possible shapes of the critical line in 2D

A notable difference between portfolio selection and reinsurance is that, while in portfolio selection the center of the system of iso-variance ellipses may lie inside or outside the set of legitimate portfolios, in reinsurance it always lies on the vertex $(0,0, \ldots, 0)$ of the legitimate set.

In the reinsurance problem, the minimum-variance portfolio is generally given (except for some special cases) by the completely reinsured portfolio, whose variance is null. Instead, in the portfolio selection problem the variance of the minimum-variance portfolio may be different from zero. "Markowitz had the brilliant insight that, while diversification would reduce risk, it would not generally eliminate it." [Rubinstein (2002, p. 1042]. This depends on covariances but also on the budget constraint, which is present in Markowitz's problem but not in de Finetti's.

Let us now give a look at the solution of the reinsurance problem reported in Figure 6. If there were no bounds on portfolio weights, all the efficient portfolios would lie on the pure critical line. Instead, in the quadratic programming problem à la de Finetti, the solution must be found in the legitimate set represented by the square BCSL. In this case, the efficient portfolios lie on the critical line, the broken line CRL.

Markowitz (2006) shows that the possible paths from $(1,1)$ to $(0,0)$ have one of the five shapes reported in Figure 7. The critical lines (a), (b), (d), (e) are made up of 2 segments while the critical 


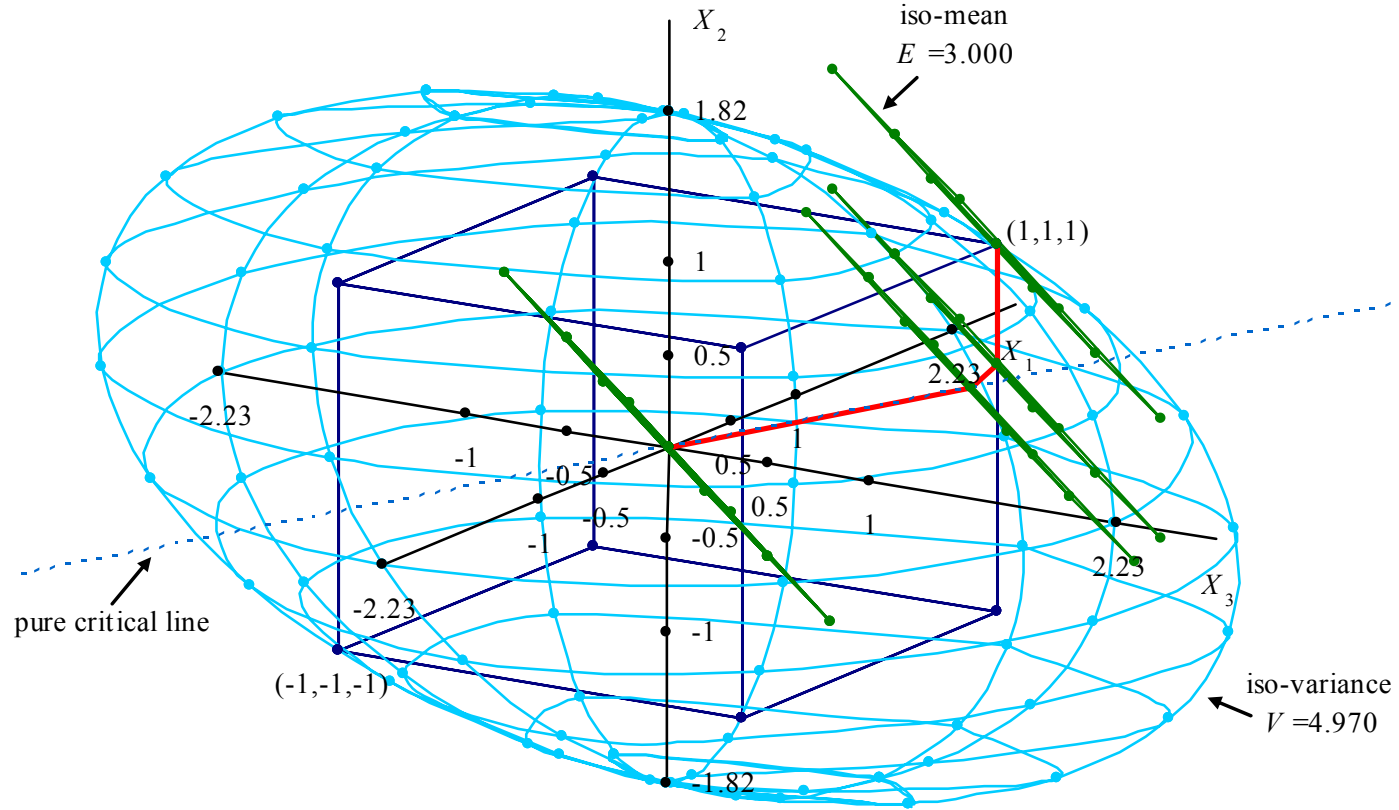

Figure 8 Efficient portfolios in the space $\left\{X_{1}, X_{2}, X_{3}\right\}: \mu_{1}=\mu_{2}=\mu_{3}=1, \sigma_{1}=\sigma_{3}=1, \sigma_{2}=\sqrt{1.5}, \rho_{12}=0.6, \rho_{13}=\rho_{23}=0$

line (c) is made up of only one segment (the degenerate case). The critical line's last segment may lie inside the square [Figure 7 (b), (c) and (d)] or on one of its boundaries [Figure 7 (d) and (e)].

The pure critical line may or may not pass through the set of legitimate portfolios. By using Markowitz's personification, we could say that the traveler tries to find the shortest route to the pure critical line but, since he has to observe the constraints, he does not necessarily reach it. This is an important point to which we will return later (Section 5, p. 17).

\section{Three Assets}

If we extend the analysis to three correlated policies, whose rates of return have means $\mu_{1}=\mu_{2}=\mu_{3}=$ 1 , standard deviations $\sigma_{1}=\sigma_{3}=1, \sigma_{2}=\sqrt{1.5}$ and correlation coefficients $\rho_{12}=0.6, \rho_{13}=\rho_{23}=0$, the reinsurance problem appears as in Figure 8.

The orientation of the ellipsoid is now negative, when we look at it from the same standpoint of Figure 3, because of the positive correlation between the first two policies. ${ }^{12}$

As far as the solution of the problem is concerned, de Finetti notes that the extension from uncorrelated to correlated assets is not difficult even if it becomes fairly complex to see the problem geometrically: ${ }^{13}$

"Let us now consider the general case in which the risks are instead correlated. It is not difficult to extend the treatment applied so far to such a case, because we only have to take into consideration the cross terms in the expression for $\sigma . "$

\footnotetext{
${ }^{12}$ In the same way as Figure 3, Figure 8 shows all the iso-mean planes corresponding to the corner portfolios but - to avoid too many lines - only the outer iso-variance ellipsoid.

${ }^{13}$ In the following citation, de Finetti changes notation to deal with the number of "dollars" (or other monetary units), $C_{h}$, to be retained for each policy, rather than with the fraction, $a_{h}$, of the policy to be retained. Besides, he refers to the $n$ hyperplanes $K_{h}$, whose intersection $\left(K_{1}=K_{2}=\ldots=K_{n}\right)$ is the pure critical line.
} 


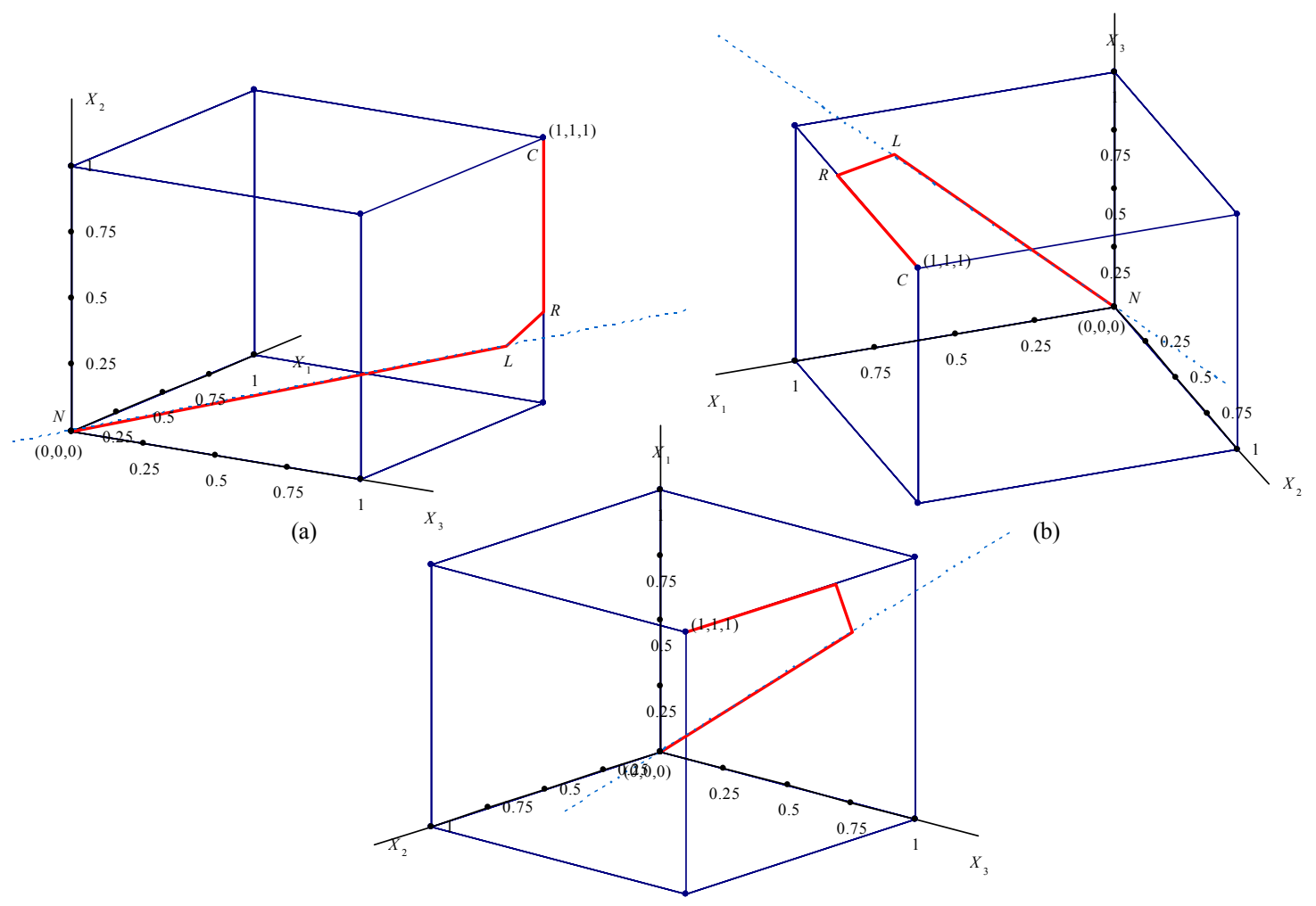

Figure 9 The critical line in 3D (correlation case)

"... the situation becomes fairly complex ... due to the analytical fact that the main axes of the ellipsoid $\sigma=$ const. are not the coordinate axes any longer, and therefore the hyperplanes $K_{h}=$ const. are not parallel to the coordinate hyperplanes. This is not a simple matter of reference that we can get around by changing the coordinates, because the reference system for the axes $C_{h}$ is tied to the intrinsic nature of the problem, since the axes constitute the edges of the hypercube of legitimate points. If we try to use new axes (the ellipsoid's main axes) as axes of reference, in order to simplify the expression for $\sigma$ by eliminating the rectangular terms, we pay the price of complicating matters by having to deal with a hypercube that is now in an oblique position."

Anyway, the solution of the problem is given again by the critical line, the broken line that links the unit point $(1,1, \ldots, 1)$ [or $\left(\bar{C}_{1}, \bar{C}_{2}, \ldots, \bar{C}_{n}\right)$, see footnote 13$]$ to the origin $(0,0, \ldots, 0)$ :

"The geometric interpretation given for the no-correlation case still holds, since the line of "optimal" points of $\sigma$ for given $G$ is a continuous broken line which links the starting point $\left(\bar{C}_{1}, \bar{C}_{2}, \ldots, \bar{C}_{n}\right)$ to the origin $(0,0, \ldots, 0)$."

The critical line of our example, observed from three standpoints, is shown as CRLN in Figure 9. ${ }^{14}$

When Markowitz (2006, p. 5) states that "De Finetti did not solve [italics in the original] the problem of computing mean-variance efficient reinsurance frontiers with correlated risks" he is probably referring to the fact that de Finetti (pp. 30-3), before moving on to examine two special cases, outlined a general procedure for dealing with correlated risks (observing that "the determination of the levels of full-risk insurances entails the laborious, though per se elementary, problem of solving a system of linear equations.") but did not work out a neat quadratic programming algorithm.

${ }^{14}$ The standpoints are $\left(10^{\circ}, 59^{\circ}, 16^{\circ}\right),\left(237^{\circ}, 180^{\circ}, 21^{\circ}\right)$ and $\left(13^{\circ}, 50^{\circ}, 105^{\circ}\right)$, respectively. 


\section{ANALYTICAL APPROACH}

We have already pointed out that Markowitz studies how to select efficient portfolios by investing a unit of capital, while de Finetti considers a given (possibly inefficient) portfolio and studies how to revise its weights, by selling (i.e. reinsuring) some of the securities (i.e. insurance policies), in order to obtain efficient portfolios. Therefore, both de Finetti and Markowitz study how to find the optimal weights $\left(a_{1}, a_{2}, \ldots, a_{n}\right.$ in de Finetti's notation, $X_{1}, X_{2}, \ldots, X_{n}$ in Markowitz's notation) of efficient portfolios.

There are two other differences between the de Finetti and Markowitz approaches:

1. in the Markowitz model, $r_{i}$ is the rate of return per dollar invested, while in the de Finetti model $X_{h}$ represents the after-reinsurance profit on the retained part of the $h^{\text {th }}$ policy, i.e. $X_{h}$ is measured in absolute terms (number of "dollars" or other monetary units) rather than in relative terms. To make the two approaches comparable, we therefore assume that each policy in the initial portfolio has a unit value, so that the whole portfolio of $n$ policies has a value equal to $n$ (in de Finetti's notation, $\bar{c}=n) ;^{15}$

2. the problem examined by de Finetti requires the minimization of portfolio variance for a given level of expected capital, not expected return. However, if the initial capital, $G_{0}$, is null (as we have generally supposed), the terms return and capital are interchangeable.

Under the above hypotheses $\left(\bar{c}=n\right.$ and $\left.G_{0}=0\right)$, the symbol used by Markowitz for denoting the portfolio's expected return, $E$, is equal to the symbol used by de Finetti, $G$.

In both the problems, the goal is to minimize the portfolio's variance, $V$, for a given expected rate of return, $E=E_{0}$. For reasons that will become evident immediately, it is convenient to minimize $V / 2$ instead of $V$ (and this obviously leads to the same solution). ${ }^{16}$ In other terms, the constrained optimization problem is:

$$
\begin{gathered}
\operatorname{minimize} \quad 1 / 2 V=1 / 2 \sum_{i=1}^{n} \sum_{j=1}^{n} X_{i} X_{j} \sigma_{i j} \\
\text { subject to } E=\sum_{i=1}^{n} X_{i} \mu_{i}=E_{0}
\end{gathered}
$$

We can write the Lagrange function as

$$
\begin{aligned}
L & =1 / 2 V-\lambda_{E}\left(E-E_{0}\right) \\
& =1 / 2 \sum_{i=1}^{n} \sum_{j=1}^{n} X_{i} X_{j} \sigma_{i j}-\lambda_{E}\left(\sum_{i=1}^{n} X_{i} \mu_{i}-E_{0}\right)
\end{aligned}
$$

\footnotetext{
${ }^{15}$ A "translation" of de Finetti's symbols into those used by Markowitz is reported in the Appendix (p. 27). In particular, the hypothesis that the portfolio of $n$ policies has a value equal to $n$ corresponds, in de Finetti's notation, to the hypothesis that $\bar{c}=n$, where $c$ is the portfolio's expected profit before reinsurance. In fact, in the world with zero risk-free interest rates implicitly assumed by de Finetti, the portfolio's value, $n$, is equal to the portfolio's expected profit, $\bar{c}$.

We also assume that reinsurance does not entail transaction costs (management expenses in de Finetti's words). Therefore, the expected loss, $\lambda_{h}$, from reinsuring the quota $\left(1-a_{h}\right)$ of the $h^{\text {th }}$ policy is equal to the expected profit, $\left(1-a_{h}\right) c_{h}$, from retaining the quota $\left(1-a_{h}\right)$ of the $h^{\text {th }}$ policy. In other terms, $\lambda_{h}=c_{h}$.

Initially, for sake of generality, de Finetti had defined $\underline{c}_{h}$ as a generic function of $a_{h}\left[c_{h}=c_{h}\left(a_{h}\right)\right]$. Later, he implicitly assumes that $c_{h}$ changes linearly with $a_{h}$ (in particular $c_{h}=a_{h} \bar{c}_{h}$, where $\bar{c}_{h}$ is the before-reinsurance expected profit on the $h^{\text {th }}$ policy). In fact:

"To express the problem's solution in explicit form, ... we only have to specify the dependence of $G\left[=\sum c_{h}\right.$ if $G_{0}=$

$0]$ on $a_{1}, a_{2}, \ldots, a_{n}$. ... Ignoring, as we want to do initially, the management expenses caused by the reinsurance, such a dependence will simply be linear."

${ }^{16}$ The division by 2 makes it possible to simplify Equation (4).
} 
The first-order conditions for minimizing $L$ are

$$
\begin{gathered}
\frac{\partial L}{\partial X_{i}}=\sum_{j=1}^{n} X_{j} \sigma_{i j}-\lambda_{E} \mu_{i}=0 \quad(i=1,2, \ldots, n) \\
\frac{\partial L}{\partial \lambda_{E}}=-\left(\sum_{i=1}^{n} X_{i} \mu_{i}-E_{0}\right)=0
\end{gathered}
$$

Equation (4) gives the Lagrange multiplier, $\lambda_{E}$, as

$$
\lambda_{E}=\frac{\sum_{j=1}^{n} X_{j} \sigma_{i j}}{\mu_{i}} \quad(i=1,2, \ldots, n)
$$

which is equal to

$$
\lambda_{E}=1 / 2 \frac{\partial V}{\partial X_{i}}: \frac{\partial E}{\partial X_{i}} \Rightarrow \lambda_{E}=1 / 2 \frac{\partial V}{\partial E}
$$

\section{Uncorrelated Assets}

When the rates of return are uncorrelated $\left(\sigma_{i J}=0\right.$ for $\left.i \neq j\right)$, Equation (6) becomes

$$
\lambda_{E}=\frac{X_{i} \sigma_{i}^{2}}{\mu_{i}} \Rightarrow X_{i}=\lambda_{E} \frac{\mu_{i}}{\sigma_{i}^{2}} \quad(i=1,2, \ldots, n)
$$

We are now ready to look again at a basic paragraph of the 1940 paper for the no-correlation case. ${ }^{17}$ In de Finetti's words (and symbols): ${ }^{18}$

"If we do not consider the constraints $0 \leq a_{h} \leq 1\left[0 \leq X_{i} \leq 1\right]$, the solution of the analytical problem is given by the straight line (passing through the origin) which is the locus of all tangencies between ellipsoids and hyperplanes. This straight line can be defined by setting equal to a constant $A$ (constant in relation to $h)\left[\lambda_{E}\right.$ (constant in relation to $i$ )] the $n$ ratios of partial derivatives

or, since

$$
\frac{\partial \sigma^{2}}{\partial a_{h}}: \frac{\partial G}{\partial a_{h}},\left[\frac{\partial V}{\partial X_{i}}: \frac{\partial E}{\partial X_{i}}\right]
$$

$$
\frac{\partial G}{\partial a_{h}}=\bar{k}_{h} \quad, \quad \frac{\partial \sigma^{2}}{\partial a_{h}}=2 a_{h} \bar{\sigma}_{h}^{2},\left[\frac{\partial E}{\partial X_{i}}=\mu_{i} \quad, \quad \frac{\partial V}{\partial X_{i}}=2 X_{i} \sigma_{i}^{2}\right]
$$

by setting $a_{h}=A \overline{k_{h}} / \bar{\sigma}_{h}^{2}\left[X_{i}=\lambda_{E} \mu_{i} / \sigma_{i}^{2}\right]$."

In the constrained optimization problems, with no (lower or upper) bounds on the variables, the firstorder condition for a maximum or minimum simply requires that the first partial derivatives of the Lagrange function with respect to all the variables and the Lagrange multipliers be zero. In the quadratic programming problem the variables' bounds come into play:

\footnotetext{
${ }^{17}$ In this paragraph, de Finetti speaks to readers acquainted with the method of Lagrange multipliers or with the total-differential approach [see Chiang (1974, p. 379)]. Daboni and Pressacco (1987, p. 255) note: “... de Finetti offers a (rather involved) proof ...”.

${ }^{18}$ Note that in the last row, where $A$ is implicitly defined, de Finetti embeds the constant $1 / 2$ in the constant $A$.
} 
"However, to make this criterion applicable, we need to make the multiplicative constant $A\left[\lambda_{E}\right]$ so small that no of the $a_{h}\left[X_{i}\right]$ is larger than unity. In other words, we should reinsure all the contracts except for the smallest (more precisely: except that - or those - corresponding to the smallest $\bar{k}_{h} / \bar{\sigma}_{h}^{2}$ ratio $\left[\mu_{i} / \sigma_{i}^{2}\right.$ ratio]), that is we should level all the risks on the basis of the smallest one. Since we can exclude - under normal conditions arising in practice - that the reinsurance has to be pushed to such a point, what remains important is to see what is the role played by the constraints $0 \leq a_{k} \leq 1\left[0 \leq X_{i} \leq 1\right]$, which we have not considered yet. The solution is the same as before but now it is necessary to set equal to 1 all the $a_{h}$ $\left[X_{i}\right]$ that would be larger than 1 .

It would be easy to see, immediately and directly, even in the case where the constant $A$ is not so small as to make all the $a_{h} \leq 1$, that the solution is the same as before but now it is necessary to set equal to 1 all the $a_{h}$ that would be larger than 1."

In particular,

"It is clear that the procedure ... reduces to considering the $a_{h}\left[X_{i}\right]$ defined by the relationship $a_{h}=A \bar{k}_{h} / \bar{\sigma}_{h}^{2}$ $\left[X_{i}=\lambda_{E} \mu_{i} / \sigma_{i}^{2}\right]$, except that we replace the values that are greater than 1 by $a_{h}=1\left[X_{i}=1\right]$, and to decreasing the constant $A\left[\lambda_{E}\right]$ from the value for which all the $a_{h}\left[X_{i}\right]$ are set equal to 1 until they all become lower than unity, and then to keep going till they reach zero."

\section{Correlated Assets}

At the time he wrote the paper on full-risk insurances, de Finetti was working for the most important Italian insurance company (Assicurazioni Generali in Trieste) and was aware of the merits of diversification. However, he was also aware that, during crises, all correlations tend to move towards 1.

In 1937 he published a paper on "About correlation", where he presents a "geometric interpretation that helps to make the relationships among mathematical expectations, standard deviations and correlation coefficients intuitive". ${ }^{19}$

In the 1940 paper he claims credit for the originality of his arguments on correlated risks:

"The extension, which seems so obvious to me, of the previous considerations on risk to the case of correlated events is almost never discussed in the research on this subject (to be more precise, I remember having seen it only in a paper by Dubois ... who however didn't address the problem of full-risk insurances)."

In the general case of correlated risks, we have already seen that the first-order conditions for minimizing the Lagrange function, $L$, are given by the system of equations (4)-(5). In matrix notation, the constrained optimization problem is as follows:

$$
\operatorname{minimize} \quad L=1 / 2 X^{\prime} C X-\lambda_{E}\left(\mu^{\prime} X-E_{0}\right)
$$

where $C$ is the variance-covariance matrix and $\mu^{\prime}$ is the return vector. The first-order conditions are

$$
\begin{gathered}
\frac{\partial L}{\partial X}=X^{\prime} C-\lambda_{E} \mu^{\prime}=0 \\
\frac{\partial L}{\partial \lambda_{E}}=-\left(\mu^{\prime} X-E_{0}\right)=0
\end{gathered}
$$

By solving the system (10) with respect to $X$, we get the equation for the pure critical line:

$$
X^{\prime}=\lambda_{E} \mu^{\prime} C^{-1}
$$

\footnotetext{
${ }^{19}$ Linear combinations of random numbers are interpreted as vectors in an "abstract space" and the correlation coefficient between random numbers $X$ and $Y$ is seen as the cosine of the "angle" between the two vectors representing $X$ and $Y$.
} 
In the case of correlated assets, de Finetti changes notation (see footnote 13) and deals with "notreinsured capitals", $C_{h}$, instead of portfolio weights, $a_{h}$ (showing his results in absolute terms rather than in relative terms). He states that the pure critical line

"... can be found by equalizing the $n$ linear functions $K_{h}$, that is by solving the system of $n$ linear equations $K_{1}\left(C_{1} \ldots C_{n}\right)=K_{2}\left(C_{1} \ldots C_{n}\right)=\ldots=K_{n}\left(C_{1} \ldots C_{n}\right) . G\left(C_{1} \ldots C_{n}\right)=G=$ given const."

Differently from the case of no correlation, de Finetti does not derive an explicit expression, such as (12), for portfolio weights, but simply states that the pure critical line can be found by solving a system of linear equations. In other terms, he does not solve the system of linear equations.

However he does give a "generic outline of the procedure" and identifies some of the properties of the solution. In particular, de Finetti points out three differences with respect to the no-correlation case:

1. "we cannot take for granted that the order $K_{1}, K_{2}, \ldots, K_{n}$ in which we subsequently meet the contracts to be reinsured is the same order as that in which the $K$ would appear in decreasing order at the initial point. Therefore, the determination of such an order is not immediate ex ante.";

2. "it will no longer be true that, along every segment of the broken line, we have to proceed in equal proportion to the reinsurance of the different reinsured contracts.";

3. "the levels of full-risk insurances are interdependent, in the sense that the ratio between two fullrisk insurances, which - in the case of no-correlation - depended only on elements of the same insurances ... may now be modified by the presence of a third risk or other additional risks, correlated with the first two to a greater or lesser extent.".

Before moving on to the analysis of two special cases, de Finetti concludes:

"It is precisely because of this circumstance [the interdependency] that, as we have seen, the determination of the levels of full-risk insurances entails the laborious, though per se elementary, problem of solving a system of linear equations."

\section{LAST SEGMENT}

One controversial issue of de Finetti's paper regards the critical line's last segment, i.e. the segment that leads to the minimum-variance efficient portfolio:

"While de Finetti does not solve the reinsurance problem with correlated risks, he outlines some of its properties. He tells where the efficient set starts; how it traces out a sequence of connected straight line segments; and then describes how it ends, i.e., the general location of the last segment of the path. I will refer to de Finetti's statement on the latter matter as 'de Finetti's last segment conjecture.' It is not correct." [Markowitz (2006, p. 6)]

Markowitz (2006) pointed out that - when returns are correlated - the last segment does not necessarily lie on the pure critical line. Instead, de Finetti writes (for the correlation case) that

“... until we will finally proceed on the last segment inside the hypercube, along the straight line $K_{1}=K_{2}$ $=\ldots=K_{n}$ ” [in Italian: “... finché finalmente si percorrerà l'ultimo tratto all'interno dell'ipercubo lungo la retta $\left.K_{1}=K_{2}=\ldots=K_{n}{ }^{\prime \prime}\right]$

There is no doubt that, in the above geometrical representation, de Finetti locates the last segment inside the hypercube and along the pure critical line.

It could be argued that de Finetti's statement does not implicitly exclude the possibility of the last segment lying on the hypercube:

1. the adverb "inside" surely rules out the points outside the hypercube but it does not necessarily exclude the points on the edges of the hypercube;

2. the adverb "along" does not rule out the possibility of the last segment touching the pure critical line at the origin, through which the pure critical line must always pass. 
However, the most reasonable justifications seem to be the following: ${ }^{20}$

1. the cases in which all the policies need to be reinsured is unlikely to be important from a practical standpoint, even if the academic relevance of the last segment (which leads to the risk-free portfolio) is undeniable. The following de Finetti's statement gives support to this interpretation:

“... all these equalities would be satisfied only when all the contracts needed to be reinsured, a point that is out of the question in practice."

2. even if we were interested in the last segment from a practical standpoint, the circumstances under which de Finetti's statement is not correct are relatively rare.

We will now review the last point, that is we will examine the cases where the critical line's last segment is not located strictly inside the legitimate set but on one of its boundaries.

The necessary (and sufficient) condition for the last segment to lie on one of the edges of the hypercube when $n=2$ has been derived by Markowitz (2006). The necessary (but not sufficient) condition for $n=3$ has been given by Pressacco (2005). A generalization for the case of $n$ dimensions has been given by Markowitz (2006, Technical Supplement).

\section{Markowitz's 2D Analysis}

Markowitz (2006) shows that, for the case of two correlated assets, the possible shapes of the critical line are those reported in Figure 7.

Figure $7 \mathrm{a}$ and Figure $7 \mathrm{~b}$ show the cases where the last segment lies strictly inside the legitimate set, while Figure 7d and Figure 7e show the cases where the last segment lies on one of its boundaries.

\section{First Segment}

Figure $7 \mathrm{c}$ shows a degenerate case, where "both $X_{1}$ and $X_{2}$ change from being UP [i.e. $X_{i}=1.0$ ] at $(1,1)$ to being IN [i.e. $\left.0<X_{i}<1.0\right]$ on the efficient segment that traverses the interior of the square" [Markowitz (2006, p. 10)].

Therefore, Figure $7 \mathrm{c}$ shows that the first segment of the critical line may lie inside the square (hypercube) and not on one of its edges, as reported in the general outline of de Finetti's procedure:

"[the] continuous broken line ... moves at first on an edge of the hypercube $0 \leq a_{h} \leq 1 \ldots$ "

The degenerate cases could have been ruled out by de Finetti if - in the following sentence - he had numbered the contracts by using the symbol " $>$ " instead of the symbol " $\geq$ ": 21

"Let us suppose we have numbered the contracts so that $\bar{\sigma}_{1}^{2} / \bar{k}_{1} \geq \bar{\sigma}_{2}^{2} / \bar{k}_{2} \geq \ldots \geq \bar{\sigma}_{n}^{2} / \bar{k}_{n} \ldots$ "

\section{Special Cases}

In the case of correlated assets, it is possible for portfolio variance to become null without the point $(0,0)$ ever being reached.

If $n=2$ and $\sigma_{1}=\sigma_{2}, \rho_{12}=-1$, the minimum-variance portfolio is given by the point $(1,1)$. In this case the critical line's last segment is given by only one point (Figure 10a).

If $n=2$ and $\sigma_{1} \neq \sigma_{2}, \rho_{12}=-1$, the minimum-variance portfolio lies on one of the edges $\left(X_{1}, 1\right)$ or $\left(1, X_{2}\right)$. In this case the critical line's last segment coincides with the first segment (Figure 10b, c). ${ }^{22}$

\footnotetext{
${ }^{20}$ See Pressacco (2005).

${ }^{21}$ Generally (and always in the no-correlation case), the strict inequality would have ruled out the possibility of degenerate cases. We considered the use of " $\geq$ " instead of ">" as a typo. See Appendix, page 28.

${ }^{22}$ For instance, when $\sigma_{1}=1, \sigma_{2}=2, \rho_{12}=-1$, any portfolio with $X_{1}=2 X_{2}$ has zero variance [Markowitz (2006, p. 16)]. In this case, the iso-variance ellipse collapses to a straight line. Moreover, when $X_{1}=1$ then $X_{2}=0.5$ : this is the end point of the critical line, whose shape is similar to that in Figure 10c.
} 


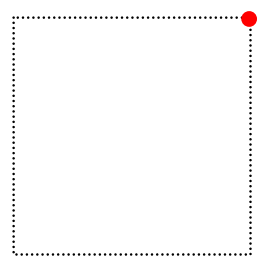

(a)

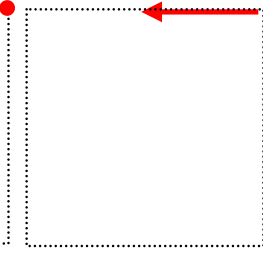

(b)

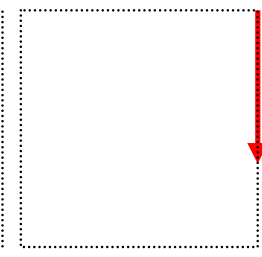

(c)

Figure 10 Possible shapes of the critical line in 2D: special cases

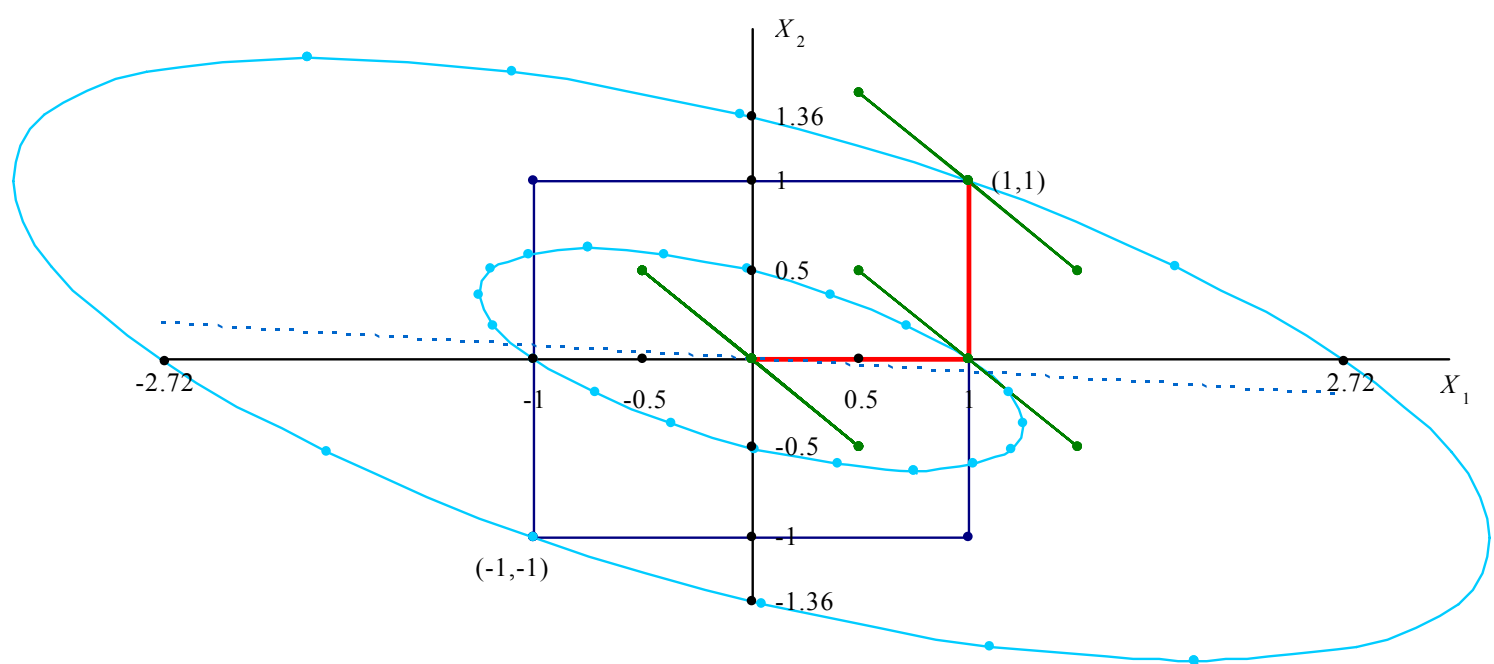

Figure 11 Efficient portfolios in the space $\left\{X_{1}, X_{2}\right\}: \mu_{1}=1, \mu_{2}=1, \sigma_{1}=1, \sigma_{2}=2, \rho_{12}=0.6$

\section{Edge Solutions}

Markowitz (2006, p. 9) highlights that

“... in the de Finetti model with correlated risks, unlike the case with uncorrelated risks, it is possible for the last segment to approach the zero portfolio along the edge of the square or along the face or edge of the cube or hypercube, rather than through its interior".

In particular, he shows that we have an edge solution of the kind shown in Figure $7 \mathrm{~d}$ if and only if

$$
\frac{\mu_{2}}{\sigma_{2}} \leq \rho_{12} \frac{\mu_{1}}{\sigma_{1}}
$$

When inequality (13) holds, the insurer has to reinsure the second policy completely before starting to reinsure the first. ${ }^{23}$

The example considered by Markowitz $\left(\mu_{1}=1, \mu_{2}=1, \sigma_{1}=1, \sigma_{2}=2, \rho_{12}=0.6\right)$ clearly shows that when inequality (13) is satisfied for an edge solution

$$
\frac{\mu_{2}}{\sigma_{2}} \leq \rho_{12} \frac{\mu_{1}}{\sigma_{1}} \text { i.e. } \frac{1}{2} \leq 0.6 \frac{1}{1}
$$

the critical line's last segment lies on the bottom edge of the legitimate square (Figure 11).

${ }^{23}$ If $\mu_{1} \geq 0, \mu_{2} \geq 0$ inequality (13) can only be satisfied by positive values of $\rho_{12}$. 
Analogously, we have an edge solution of the kind shown in Figure 7e if and only if

$$
\frac{\mu_{1}}{\sigma_{1}} \leq \rho_{12} \frac{\mu_{2}}{\sigma_{2}}
$$

When $\mu_{1} \geq 0, \mu_{2} \geq 0, \rho_{12}=0$, edge solutions are ruled out since inequalities (13) and (15) can never hold.

\section{Internal Solutions}

Markowitz's proof of (13) is based on the signs of the first-order derivatives of the Lagrange function with respect to portfolio weights, measured at the point $\left(X_{1}=1, X_{2}=0\right)$.

Another approach has been followed by Pressacco (2005), who, by solving (12) for $n=2$, obtains the pure critical line as: ${ }^{24}$

$$
X_{2}=\frac{\mu_{2} \sigma_{1}^{2}-\mu_{1} \sigma_{12}}{\mu_{1} \sigma_{2}^{2}-\mu_{2} \sigma_{12}} X_{1}
$$

Then, by requiring the slope of the pure critical line (16) to be positive, Pressacco derives the internality condition for the last segment as: ${ }^{25}$

$$
\rho_{12}<\frac{\mu_{1} / \sigma_{1}}{\mu_{2} / \sigma_{2}}
$$

with the implicit assumption that $\left(\mu_{1} / \sigma_{1}\right)<\left(\mu_{2} / \sigma_{2}\right){ }^{26}$

Inequality (17) is "complementary" to inequality (15). When the slope of the pure critical line is negative, the reinsurance problem always has an edge solution, as in Figure 11. However, it is not true that when the slope of the pure critical line is positive, the reinsurance problem always has an internal solution. In other terms, inequality (17) is a necessary but not sufficient condition for an internal solution. This is due to the fact that, when returns are correlated, the zero-variance portfolio can be reached before getting to the origin.

\section{Pressacco's 3D Extension}

Pressacco extends his analysis to three dimensions, looking for the conditions necessary to guarantee an internal solution to the reinsurance problem.

By solving (12) for $n=3$, it is possible to obtain the pure critical line, $X^{\prime} \equiv\left[X_{1} X_{2} X_{3}\right]$, as a function of $\lambda_{E}{ }^{27}$ and the bilateral relationships between $X_{1}, X_{2}$ and $X_{3}$, that is the projections of the pure critical line on the cube's faces $\left\{X_{1}, X_{2} \mid X_{3}=0\right\},\left\{X_{1}, X_{3} \mid X_{2}=0\right\}$ and $\left\{X_{2}, X_{3} \mid X_{1}=0\right\}$ :

\footnotetext{
${ }^{24}$ Equation (16) can be obtained from (12) by noting that

$$
C^{-1}=\frac{1}{|C|} \text { adj } C=\frac{1}{\sigma_{1}^{2} \sigma_{2}^{2}-\sigma_{12}^{2}}\left[\begin{array}{cc}
\sigma_{2}^{2} & -\sigma_{12} \\
-\sigma_{12} & \sigma_{1}^{2}
\end{array}\right]
$$

${ }^{25}$ In his notation, $r<z / t$, with $t=m_{y} \sigma_{x}$ and $z=m_{x} \sigma_{y}$. To obtain (17), substitute $r$ with $\rho_{12}, m_{y} \sigma_{x}$ with $\mu_{2} \sigma_{1}$ and $m_{x} \sigma_{y}$ with $\mu_{1} \sigma_{2}$.

${ }^{26}$ Inequality (17) has been derived by requiring the slope's denominator in (17) to be greater than zero. For the slope to be positive, the numerator also has to be positive. Therefore, $\mu_{2} \sigma_{1}-\mu_{1} \sigma_{12}=\sigma_{1}\left(\mu_{2} \sigma_{1}-\mu_{1} \sigma_{2} \rho_{12}\right)>0 \Rightarrow \mu_{2} \sigma_{1}>\mu_{1} \sigma_{2} \rho_{12} \Rightarrow \rho_{12}<\left(\mu_{2} / \sigma_{2}\right) /\left(\mu_{1} / \sigma_{1}\right)$. To be sure that this inequality is satisfied we have to set $\left(\mu_{1} / \sigma_{1}\right)<\left(\mu_{2} / \sigma_{2}\right)$, so that the ratio $\left(\mu_{2} / \sigma_{2}\right) /\left(\mu_{1} / \sigma_{1}\right)$ is greater than 1 .

${ }^{27}$ To solve (12) we first calculate $C^{-1}$ as$$
C^{-1}=\frac{1}{|C|} \text { adj } C=\frac{1}{\sigma_{1}^{2} \sigma_{2}^{2} \sigma_{3}^{2}+2 \sigma_{12} \sigma_{13} \sigma_{23}-\sigma_{1}^{2} \sigma_{23}^{2}-\sigma_{2}^{2} \sigma_{13}^{2}-\sigma_{3}^{2} \sigma_{12}^{2}}\left[\begin{array}{ccc}
\sigma_{2}^{2} \sigma_{3}^{2}-\sigma_{23}^{2} & \sigma_{12} \sigma_{13}-\sigma_{3}^{2} \sigma_{12} & \sigma_{12} \sigma_{23}-\sigma_{2}^{2} \sigma_{13} \\
\sigma_{12} \sigma_{13}-\sigma_{3}^{2} \sigma_{12} & \sigma_{1}^{2} \sigma_{3}^{2}-\sigma_{13}^{2} & \sigma_{12} \sigma_{13}-\sigma_{1}^{2} \sigma_{23} \\
\sigma_{12} \sigma_{23}-\sigma_{2}^{2} \sigma_{13} & \sigma_{12} \sigma_{13}-\sigma_{1}^{2} \sigma_{23} & \sigma_{1}^{2} \sigma_{2}^{2}-\sigma_{12}^{2}
\end{array}\right]
$$ 


$$
\begin{aligned}
& X_{2}=\frac{\mu_{1}\left(\sigma_{13} \sigma_{23}-\sigma_{3}^{2} \sigma_{12}\right)+\mu_{2}\left(\sigma_{1}^{2} \sigma_{3}^{2}-\sigma_{13}^{2}\right)+\mu_{3}\left(\sigma_{12} \sigma_{13}-\sigma_{1}^{2} \sigma_{23}\right)}{\mu_{1}\left(\sigma_{2}^{2} \sigma_{3}^{2}-\sigma_{23}^{2}\right)+\mu_{2}\left(\sigma_{13} \sigma_{23}-\sigma_{3}^{2} \sigma_{12}\right)+\mu_{3}\left(\sigma_{12} \sigma_{23}-\sigma_{2}^{2} \sigma_{13}\right)} X_{1} \\
& X_{3}=\frac{\mu_{1}\left(\sigma_{12} \sigma_{23}-\sigma_{2}^{2} \sigma_{13}\right)+\mu_{2}\left(\sigma_{12} \sigma_{13}-\sigma_{1}^{2} \sigma_{23}\right)+\mu_{3}\left(\sigma_{1}^{2} \sigma_{2}^{2}-\sigma_{12}^{2}\right)}{\mu_{1}\left(\sigma_{13} \sigma_{23}-\sigma_{3}^{2} \sigma_{12}\right)+\mu_{2}\left(\sigma_{1}^{2} \sigma_{3}^{2}-\sigma_{13}^{2}\right)+\mu_{3}\left(\sigma_{12} \sigma_{13}-\sigma_{1}^{2} \sigma_{23}\right)} X_{2} \\
& X_{1}=\frac{\mu_{1}\left(\sigma_{2}^{2} \sigma_{3}^{2}-\sigma_{12}^{2}\right)+\mu_{2}\left(\sigma_{13} \sigma_{23}-\sigma_{3}^{2} \sigma_{12}\right)+\mu_{3}\left(\sigma_{12} \sigma_{23}-\sigma_{2}^{2} \sigma_{13}\right)}{\mu_{1}\left(\sigma_{12} \sigma_{23}-\sigma_{2}^{2} \sigma_{13}\right)+\mu_{2}\left(\sigma_{12} \sigma_{13}-\sigma_{1}^{2} \sigma_{23}\right)+\mu_{3}\left(\sigma_{1}^{2} \sigma_{2}^{2}-\sigma_{12}^{2}\right)} X_{3}
\end{aligned}
$$

Pressacco then obtains the internality conditions by requiring the slopes of the above equations to be positive. Finally, under the hypothesis that correlations are uniformly positive $\left(\rho_{12}=\rho_{13}=\rho_{23}=\rho\right.$ ) and after numbering the policies so that $\left(\mu_{1} / \sigma_{1}\right) \leq\left(\mu_{2} / \sigma_{2}\right) \leq\left(\mu_{3} / \sigma_{3}\right)$, he derives the following criterion for guaranteeing the internality of the critical line's last segment: ${ }^{28}$

$$
\frac{\rho}{1+\rho}<\frac{\left(\mu_{1} / \sigma_{1}\right)}{\left(\mu_{2} / \sigma_{2}\right)+\left(\mu_{3} / \sigma_{3}\right)}
$$

The above condition is necessary for the last segment to lie inside the cube and, differently from the $2 \mathrm{D}$ case, it is also sufficient. This depends on the hypothesis that correlations are uniformly positive. In such a case the zero-variance portfolios can only be built by giving zero weights to all the policies and the critical line always ends at the origin $(0,0,0)$.

\section{Markowitz Generalization}

Markowitz uses the Technical Supplement of his article to generalize the results in $n$ dimensions. He proves that the correct "last segment proposition" for the de Finetti model is the following:

"... as we trace out the set of efficient segments, from that with the highest mean to that with lowest variance, the first efficient segment we encounter with no UP variables is the last segment [a variable $X_{i}$ is UP if is fixed at 1.0 throughout the segment].... once we encounter an efficient segment with no variable UP, the segment heads directly towards the risk-free final portfolio."

Markowitz adds that

"If $C$ is nonsingular, then the zero vector is indeed the unique minimum variance efficient portfolio."

The hypothesis of non-singularity for $C$ rules out the special cases we have considered in the section Special Cases of Markowitz's 2D Analysis.

\section{CONCLUSIONS}

In an important 1940 paper on full-risk insurances, Bruno de Finetti tried to find answers to the following question: "How should an insurance company behave in maximizing the profit of a portfolio of insurance contracts subject to the constraint given by the risk level it is ready to bear?". In other terms: "Which contracts should it reinsure and to what extent?" This question can also be formulated in an equivalent way: "What are the optimal mean-variance weights of the portfolio that allow the insurance company to minimize the risk of default subject to the constraint given by the profit level it must reach?"

\footnotetext{
${ }^{28}$ The criterion has been corrected for a typo, by substituting the original $1-\rho$ with $1+\rho$.
} 
Bruno de Finetti gave anticipatory answers to the reinsurance problem, a special case in the class of portfolio selection problems. The original contributions of de Finetti have recently been acknowledged by the 1990 Nobel laureate Harry Markowitz, who generously titled a 2006 article as "De Finetti Scoops Markowitz". ${ }^{29}$ The two authors, de Finetti and Markowitz, did indeed walk unknowingly on similar roads at different times.

De Finetti's standpoint is partly different from the one used by Markowitz in his classical work on portfolio selection. De Finetti deals with a portfolio of $n$ securities (i.e. insurance contracts) where the initial weights are all equal to 1 and wishes to find a method to minimize the risk, for a given level of return, by reducing (possibly to zero) some weights through pro-rata reinsurance contracts "where a certain proportion of over-risky contracts is transferred to the reinsurer". The aim of Markowitz's approach is to find all the mean-variance efficient portfolios in which to invest a given amount of capital, starting from the maximum-expected-return portfolio and ending with the minimum-risk portfolio.

De Finetti's reinsurance problem and Markowitz's portfolio selection problem are special cases of a class of models solved by the critical line algorithm (CLA) provided in Markowitz (1956). By making use of this algorithm, we tested the controversial issue raised by Markowitz in his 2006 article: the critical line's last segment.

When he described the geometrical solution of the reinsurance problem for the case of correlated assets, Bruno de Finetti wrote: "... until we will finally proceed on the last segment inside the hypercube, along the straight line $K_{1}=K_{2}=\ldots=K_{n}$ ". The straight line $K_{1}=K_{2}=\ldots=K_{n}$, which we called the pure critical line, gives the solution of the problem if there are no bounds on portfolio weights. In interpreting de Finetti's words, it seems that the last segment of the efficient set must always lie on the pure critical line.

Harry Markowitz highlights that this point is not correct and shows that the correct lastsegment proposition for the de Finetti model is the following: "as we trace out the set of efficient segments, from that with the highest mean to that with lowest variance, the first efficient segment we encounter with no UP variables is the last segment [a variable $X_{i}$ is UP if is fixed at 1.0 throughout the segment]." In other terms, it does not necessarily holds that the critical line's last segment has always to lie on the pure critical line. It may actually lie on one of the edges of the hypercube.

Despite the controversy on this point, Markowitz acknowledges the merits of de Finetti's paper. He recognizes that de Finetti solved the problem of computing mean-variance efficient reinsurance frontiers for the case of uncorrelated risks and gives him credit for outlining some of the properties that characterize the solution of the reinsurance problem with correlated risks. He also acknowledges that de Finetti worked out a special case of the Kuhn-Tucker theorem (considered the single most important analytical result in nonlinear programming) when there are upper and lower bounds on variables but no equality constraints. Finally, he points out that de Finetti's achievements did not benefit from an environment in which "the existence of the Kuhn-Tucker theorem and the success of linear programming encouraged a presumption that a neat quadratic programming algorithm existed if we persisted in seeking it."

\footnotetext{
29 "Harry Markowitz first published his mean-variance portfolio selection theory in 1952, and later, in 1959, published his book Portfolio Selection, probably the most important book written in the history of financial economics. It rests firmly at the root of the next half century of research". [Rubinstein (1999), p. 324].
} 


\section{REFERENCES}

Bühlmann, Hans, Mathematical Methods in Risk Theory, Springer-Verlag, New York, Heidelberg, Berlin, 1970.

ChiAng, Alpha C., Fundamental Methods of Mathematical Economics, $2^{\text {nd }}$ ed., McGraw-Hill, 1974.

Daboni, Luciano, "Elenco degli scritti di Bruno de Finetti", in Atti del Convegno "Ricordo di Bruno de Finetti Professore nell'Ateneo triestino”, Trieste, 30-31 maggio 1986.

Daboni, Luciano and Pressacco, Flavio: "Mean Variance, Expected Utility and Ruin Probability in Reinsurance Decisions: Suggestions and Comments on the Line of de Finetti's Seminal Work", in VierTL, L. (Ed.), Probability and Bayesian Statistics, Plenum Publishing Corp., 1987.

DANTZIG, George B., "Maximization of a Linear Function of Variables Subject to Linear Inequalities", in KoOPMANS, Tjalling C. (ed.), Activity Analysis of Production and Allocation, Cowles Foundation, Monograph 16, Yale University Press, 1951. See cowles.econ.yale.edu/P/cm/m13/m13-all.pdf.

DE FInETTI, Bruno, "Problemi di “optimum"” and "Problemi di “optimum" vincolato", Giornale dell'Istituto Italiano degli Attuari, Vol. 8, No. 1-2, 1937a,b.

DE FInetTI, Bruno, “A proposito di correlazione”, Supplemento Statistico ai Nuovi problemi di Politica, Storia ed Economia, 3, 41-57, 1937c. An English version, translated by Luca Barone and Peter Laurence in 2006, appears as "About Correlation," in Insurance, Mathematics and Economics, 2007 (forthcoming).

DE FinetTi, Bruno, “II problema dei pieni”, Giornale dell'Istituto Italiano degli Attuari, Vol. 11, No. 1, pp. 1-88, 1940. An English version of the first chapter, "The Problem in a Single Accounting Period," translated by Luca Barone in 2005, appears as "The Problem of "Full-Risk Insurances"”, in Journal of Investment Management, "Special Issue: A Literature Postscript", Vol. 4, No. 3, pp. 19-43, 2006.

Elderton, William, Nicholl, Charles, SeAL, Hiliary, "Notes on Foreign Actuarial Journals", Journal of the Institute of Actuaries, Vol. 73, p. 149, 1947 ((www.actuaries.org.uk/files/pdf/library/JIA-073/0140-0158.pdf).

Lagrange, Joseph Louis, "Recherches sur la Methode de Maximis et Minimis”, Ouvres, Vol. 1, 1759.

KAHN, Paul Marham, "Book Reviews and Notices”, Transactions of Society of Actuaries, Vol. 23, Pt. 1, No. 65, pp. 59-60, 1971.

Kolmogoroff, A., Sulla forma generale di un processo stocastico omogeneo (Un problema di Bruno de Finetti), Rend. R. Acc. Naz. Lincei, vol. XV, Ser. $6^{\text {a }}, 1^{\circ}$ sem., fasc. 10 (e seconda Nota nel fasc. 11), 1932.

Markowitz, Harry M., Portfolio Selection: Efficient Diversification of Investments, Cowles Foundation, Monograph 16, Yale University Press, 1959, $4^{\text {th }}$ printing (1976). Also in cowles.econ.yale.edu/P/cm/m16/m16-all.pdf .

Markowitz, Harry M., Mean-Variance Analysis in Portfolio Choice and Capital Markets, Basil Blackwell Ltd., Oxford, 1987.

Markowitz, Harry M., "The Early History of Portfolio Theory: 1600-1960”, Financial Analysts Journal, Vol. 55, no. 4, pp. 5-16, July 1999.

Markowitz, Harry M., "De Finetti Scoops Markowitz", in Journal of Investment Management, "Special Issue: A Literature Postscript", Vol. 4, No. 3, pp. 3-18, 2006.

Markowitz, Harry M. and TodD, Peter, Mean-Variance Analysis in Portfolio Choice and Capital Markets, revised reissue of Markowitz (1987) with Chapter 13 by Peter Todd, Wiley, 2000.

Nicotra, Luca, "Bruno de Finetti: così è, se vi pare", Notizie in ... Controluce, June 2004 - February 2005.

PressacCo, Flavio, "De Finetti, Markowitz e la congettura dell'ultimo segmento", Working Paper, September $13^{\text {th }}$, 2005.

PRESSACCO, Flavio, "Separation Theorems in Proportional Insurance,", pp 209-15, in GoovAERTS, M., et al., Insurance and Risk Theory, D. Reidel Publishing, 1986.

RoY, Andrew D., "Safety First and the Holding of Assets", Econometrica, Vol. 20, No. 3, pp. 431-49, July 1952.

Rubinstein, Mark, Rubinstein on Derivatives - Futures, Options and Dynamic Strategies, Risk Books, London, 1999. 
Rubinstein, Mark, “Markowitz's 'Portfolio Selection': A Fifty-Year Retrospective”, Journal of Finance, Vol. 57, No. 3, pp. 1041-5, June 2002.

Rubinstein, Mark, "Bruno de Finetti and Mean-Variance Portfolio Selection", in Journal of Investment Management, "Special Issue: A Literature Postscript", Vol. 4, No. 3, pp. 1-2, 2006.

SCHMitTER, Hans, “Setting Optimal Reinsurance Retentions”, Swiss Reinsurance Company, 2003. 


\section{APPENDIX}

\section{Notation}

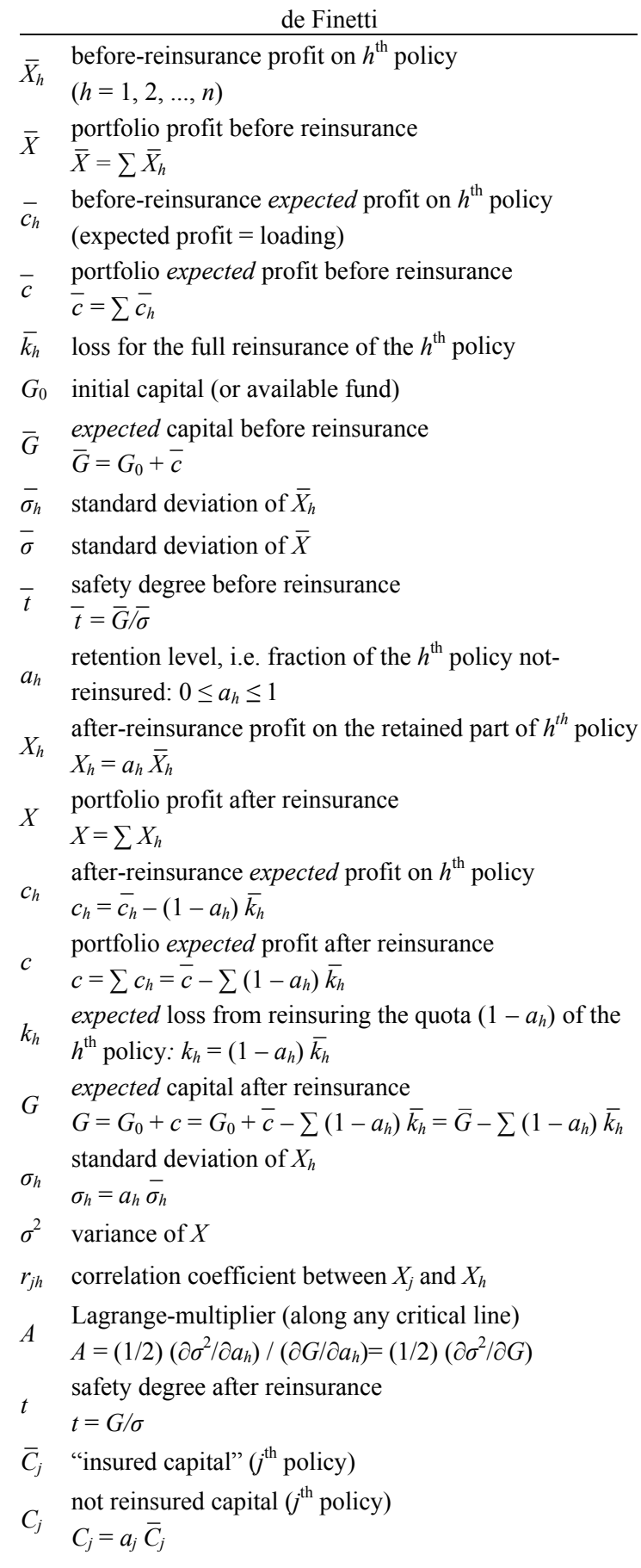

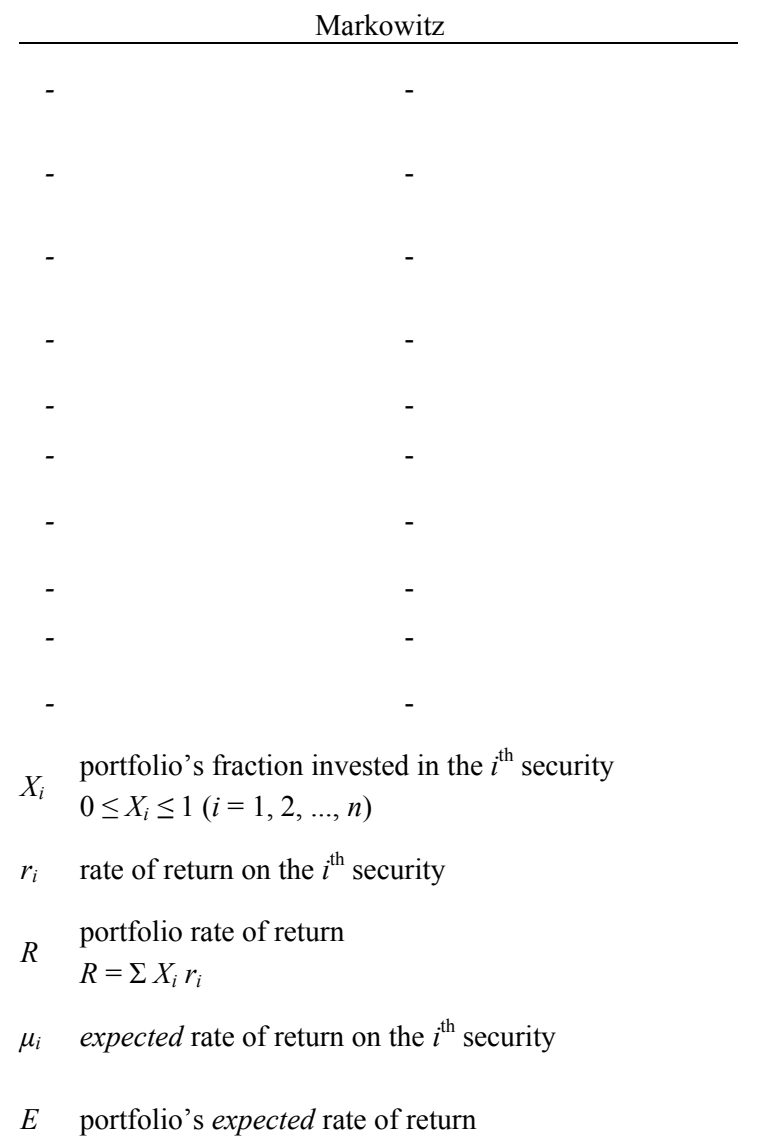

$\sigma_{i} \quad$ standard deviation of $r_{i}$

$V \quad$ variance of $R$

$\rho_{i j} \quad$ correlation coefficient between $r_{i}$ and $r_{j}$

$\lambda_{E} \quad$ Lagrange-multiplier (along any critical line) $\lambda_{E}=(1 / 2)\left(\partial V / \partial X_{i}\right) /\left(\partial E / \partial X_{i}\right)=(1 / 2)(\partial V / \partial E)$

$L \quad$ Lagrange function

$K_{h}$ derivative of the Lagrange function wit respect to $a_{h}$ 


\section{Technical Supplement}

Portfolio Selection vs. Reinsurance

\section{PORTFOLIO SELECTION}

In the portfolio selection model, an investor chooses the fractions $X_{1}, X_{2}, \ldots, X_{n}$ invested in $n$ securities. He has to minimize the variance of returns, $V$, subject to linear constraints, for every expected return, $E$ :

$$
\begin{array}{ll}
\text { minimize } & V=X^{\prime} C X \\
\text { subject to } & A X \leq b \\
& \mu^{\prime} X=E
\end{array}
$$

where

$$
\begin{gathered}
X^{\prime}=\left[X_{1}, X_{2}, \ldots, X_{n}\right] \\
0 \leq X_{i} \leq 1 \quad(i=1,2, \ldots, n) \\
C=\left[\begin{array}{cccc}
\sigma_{11} & \sigma_{12} & \ldots & \sigma_{1 n} \\
\sigma_{21} & \sigma_{22} & \ldots & \sigma_{2 n} \\
\ldots & \ldots & \ldots & \ldots \\
\sigma_{n 1} & \sigma_{n 2} & \ldots & \sigma_{n n}
\end{array}\right] \\
A=\left[\begin{array}{llll}
1 & 1 & \ldots & 1
\end{array}\right] \\
\mu^{\prime}=\left[\begin{array}{llll}
\mu_{1} & \mu_{2} & \ldots & \mu_{n}
\end{array}\right]
\end{gathered}
$$

and $V, E$ are scalars.

The inequalities in (a2) and (a5) "can be cast into the above format by introducing slack variables or by separating variables into their positive and negative parts."

\section{REINSURANCE}

In the reinsurance model, an insurer holds a portfolio of $n$ policies and chooses the fractions $Y_{1}, Y_{2}, \ldots, Y_{n}$ to sell (reinsure). He has to minimize the variance of returns, $V$, subject to linear constraints, for every expected return $E$ :

$$
\begin{aligned}
& \text { minimize } W=Y^{\prime} \Omega Y \\
& \text { subject to } B Y \leq c \\
& v^{\prime} Y=F
\end{aligned}
$$

where

$$
\begin{gathered}
Y^{\prime}=\left[Y_{1}, Y_{2}, \ldots, Y_{n}, Y_{0}\right] \\
Y_{0}=1 \quad \text { and } \quad-1 \leq Y_{i} \leq 0 \quad(i=1,2, \ldots, n) \\
\Omega=\left[\begin{array}{ccccc}
\sigma_{11} & \sigma_{12} & \ldots & \sigma_{1 n} & \sum_{j=1}^{n} \sigma_{1 j} \\
\sigma_{21} & \sigma_{22} & \ldots & \sigma_{2 n} & \sum_{j=1}^{n} \sigma_{2 j} \\
\ldots & \ldots & \ldots & \ldots & \ldots \\
\sigma_{n 1} & \sigma_{n 2} & \ldots & \sigma_{n n} & \sum_{j=1}^{n} \sigma_{n j} \\
\sum_{i=1}^{n} \sigma_{i 1} & \sum_{i=1}^{n} \sigma_{i 2} & \ldots & \sum_{i=1}^{n} \sigma_{i n} & \sum_{i=1}^{n} \sum_{j=1}^{n} \sigma_{i j}
\end{array}\right] \\
B=\left[\begin{array}{lllll}
1 & 1 & \ldots & 1 & 0
\end{array}\right] \\
v^{\prime}=\left[\begin{array}{lllll}
v_{1} & v_{2} & \ldots & v_{n} & v_{0}
\end{array}\right]
\end{gathered}
$$

and $W, F$ are scalars.

The inequalities in (a11) and (a14) "can be cast into the above format by introducing slack variables or by separating variables into their positive and negative parts."

(Note that bounds (a5) and (a14) make constraints (a2) and (a11) redundant.) 
It can be easily checked that the reinsurance problem is equivalent to the portfolio selection problem if the two following conditions are met:

1. the expected return, $v_{i}$, that is lost by reinsuring the $i^{\text {th }}$ contract is equal to the expected return, $\mu_{i}$, of the $i^{\text {th }}$ security chosen; ${ }^{30}$

2. the expected return, $v_{0}$, on the original portfolio of $n$ policies is equal to the sum of the expected returns that may be lost by reinsuring all the policies.

In other terms, the reinsurance problem and the portfolio selection problem are equivalent if reinsurance does not entail transaction costs.

In fact, the two above conditions are:

$$
v_{i}=\mu_{i} \quad(i=1,2, \ldots, n) \quad \text { and } \quad v_{0}=\sum_{i=1}^{n} v_{i}=\sum_{i=1}^{n} \mu_{i}
$$

In this case, the retention level, $Z_{i}$, defined by

$$
Z_{i}=1+Y_{i}
$$

is equal to the fraction, $X_{i}$, invested in the $i^{\text {th }}$ security. Therefore, the fraction, $Y_{i}$, of the $i^{\text {th }}$ policy to be sold (reinsured) is given by

$$
Y_{i}=X_{i}-1
$$

and the constraint (a14), $-1 \leq Y_{i} \leq 0$, is equal to the constraint (a5), $0 \leq X_{i} \leq 1$.

When (a19) and (a21) apply, constraint (a12) is equal to constraint (a3):

$$
\begin{aligned}
F & =v^{\prime} Y=\left[\begin{array}{lllll}
\mu_{1} & \mu_{2} & \ldots & \mu_{n} & \sum_{i=1}^{n} \mu_{i}
\end{array}\right] Y=\sum_{i=1}^{n} \mu_{i} Y_{i}+\sum_{i=1}^{n} \mu_{i} \\
& =\sum_{i=1}^{n} \mu_{i}\left(X_{i}-1\right)+\sum_{i=1}^{n} \mu_{i}=\sum_{i=1}^{n} \mu_{i} X_{i}=\mu^{\prime} X=E
\end{aligned}
$$

In addition, constraint (a11) is equal to constraint (a2):

$$
B Y \leq 0 \Rightarrow\left[\begin{array}{lllll}
1 & 1 & \ldots & 1 & 0
\end{array}\right]\left[\begin{array}{c}
X_{1}-1 \\
X_{2}-1 \\
\ldots \\
X_{n}-1 \\
1
\end{array}\right]=\sum_{i=1}^{n} X_{i}-n=A X-n \leq 0 \Rightarrow A X \leq n
$$

Finally, objective function (a10) is equal to objective function (a1):

$$
W=Y^{\prime} \Omega Y=\left[\begin{array}{llllll}
X_{1}-1 & X_{2}-1 & \ldots & X_{n}-1 & 1
\end{array}\right]\left[\begin{array}{cccccc}
\sigma_{11} & \sigma_{12} & \ldots & \sigma_{1 n} & \sum_{j=1}^{n} \sigma_{1 j} \\
\sigma_{21} & \sigma_{22} & \ldots & \sigma_{2 n} & \sum_{j=1}^{n} \sigma_{2 j} \\
\ldots & \ldots & \ldots & \ldots & \ldots \\
\sigma_{n 1} & \sigma_{n 2} & \ldots & \sigma_{n n} & \sum_{j=1}^{n} \sigma_{n j} \\
\sum_{i=1}^{n} \sigma_{i 1} & \sum_{i=1}^{n} \sigma_{i 2} & \ldots & \sum_{i=1}^{n} \sigma_{i n} & \sum_{i=1}^{n} \sum_{j=1}^{n} \sigma_{i j}
\end{array}\right]\left[\begin{array}{c}
X_{1}-1 \\
X_{2}-1 \\
\ldots \\
X_{n}-1 \\
1
\end{array}\right]
$$

\footnotetext{
${ }^{30}$ In de Finetti's notation, this is equivalent to setting $\lambda_{h}=c_{h}$.
} 


$$
\begin{aligned}
& =\left[\begin{array}{llll}
\sum_{i=1}^{n} X_{i} \sigma_{i 1} & \sum_{i=1}^{n} X_{i} \sigma_{i 2} \quad \ldots & \sum_{i=1}^{n} X_{i} \sigma_{i n} & \sum_{i=1}^{n} \sum_{j=1}^{n} X_{i} \sigma_{i j}
\end{array}\right]\left[\begin{array}{c}
X_{1}-1 \\
X_{2}-1 \\
\ldots \\
X_{n}-1 \\
1
\end{array}\right] \\
& =\sum_{i=1}^{n} \sum_{j=1}^{n} X_{i} X_{j} \sigma_{i j}=X^{\prime} \Omega X=V
\end{aligned}
$$

\section{Typos}

In the English translation of the paper, I did not correct the typos I found in the original article. They are listed below:

1. p. 24 , right column, $2^{\text {nd }}$ paragraph, $6^{\text {th }}$ row. Substitute $\bar{k}_{h} / \bar{\sigma}_{h}^{2}$ with $\bar{\sigma}_{h}^{2} / \bar{k}_{h}$.

2. p. 24, right column, last equation. Substitute

$$
=-2 \sum \bar{\sigma}_{h}^{2} d a_{h} ; \text { with }=-2 \sum \sigma_{h}^{2} d a_{h} ;
$$

3. page 25 , left column, first 2 rows. Replace the inequality signs $\geq$ by the strict inequality sign $>$. If $\bar{\sigma}_{1}^{2} / \bar{k}_{1}=\bar{\sigma}_{2}^{2} / \bar{k}_{2}$ this means we would have to start by reinsuring the first two contracts, not only the first.

4. p. 28, left column, first formula. Substitute

$$
C_{j}=a_{j} \bar{C}=A \frac{\left(\bar{k}_{j} / \bar{C}_{j}\right)}{\left(\bar{\sigma}_{j} / \bar{C}_{j}\right)^{2}}, \quad \text { with } \quad C_{j}=a_{j} \bar{C}_{j}=A \frac{\left(\bar{k}_{j} / \bar{C}_{j}\right)}{\left(\bar{\sigma}_{j} / \bar{C}_{j}\right)^{2}},
$$

5. p. 28 , left column, $2^{\text {nd }}$ paragraph, $2^{\text {nd }}$ line. Substitute $\bar{C}$ with $\bar{C}_{j}$

6. p. 31, right column, penultimate formula. Substitute

$$
\frac{\partial \sigma^{2}}{\partial a_{h}}: \frac{\partial G}{\partial a_{h}}=2 C_{h} \frac{\left(\bar{\sigma} / C_{h}\right)^{2}}{\left(\bar{k} / \bar{C}_{h}\right)} \text { with } \frac{\partial \sigma^{2}}{\partial a_{h}}: \frac{\partial G}{\partial a_{h}}=2 C_{h} \frac{\left(\bar{\sigma}_{h} / \bar{C}_{h}\right)^{2}}{\left(\bar{k}_{h} / \bar{C}_{h}\right)}
$$

and

$$
+2 \sum_{j}^{(h)} C_{j} r_{j h} \frac{\left(\bar{\sigma}_{j} / \bar{C}_{j}\right)\left(\bar{\sigma} / \bar{C}_{h}\right)}{\bar{k}_{h} / \bar{C}_{h}} \quad \text { with }+2 \sum_{j}^{(h)} C_{j} r_{j h} \frac{\left(\bar{\sigma}_{j} / \bar{C}_{j}\right)\left(\bar{\sigma}_{h} / \bar{C}_{h}\right)}{\bar{k}_{h} / \bar{C}_{h}}
$$

7. p. 31, right column, last formula. Substitute

$$
\frac{2 \bar{\sigma}_{h}}{\bar{k}_{h}}\left(\bar{\sigma} \frac{C_{h}}{\bar{C}_{h}}+\sum_{j}^{(h)} r_{j h} \sigma_{j} \frac{C_{j}}{\bar{C}_{j}}\right) \text { with } \frac{2 \bar{\sigma}_{h}}{\bar{k}_{h}}\left(\bar{\sigma}_{h} \frac{C_{h}}{\bar{C}_{h}}+\sum_{j}^{(h)} r_{j h} \bar{\sigma}_{j} \frac{C_{j}}{\bar{C}_{j}}\right)
$$

